Southern Illinois University Carbondale

OpenSIUC

8-14-2009

\title{
Analytic Normal Forms and Symmetries of Strict Feedforward Control Systems
}

Issa Amadou Tall

Southern Illinois University Carbondale, itall@math.siu.edu

Witold Respondek

Institut National des Sciences Appliquees de Rouen, LMI, France, wresp@insa-rouen.fr

Follow this and additional works at: http://opensiuc.lib.siu.edu/math_articles This is a preprint (prior to peer review) of the article published as Tall, I. A., \& Respondek, W. (2009). Analytic normal forms and symmetries of strict feedforward control systems. International Journal of Robust and Nonlinear Control. doi: 10.1002/rnc.1505. The definitive version is available at http://onlinelibrary.wiley.com/.

\section{Recommended Citation}

Tall, Issa A. and Respondek, Witold. "Analytic Normal Forms and Symmetries of Strict Feedforward Control Systems." (Aug 2009).

This Article is brought to you for free and open access by the Department of Mathematics at OpenSIUC. It has been accepted for inclusion in Articles and Preprints by an authorized administrator of OpenSIUC. For more information, please contact opensiuc@lib.siu.edu. 


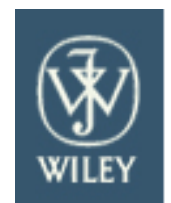

International Journal of

Robust and Nonlinear Control

\section{Analytic Normal Forms and Symmetries of Strict Feedforward Control Systems}

\begin{tabular}{|c|c|}
\hline Journal: & International Journal of Robust and Nonlinear Control \\
\hline Manuscript ID: & draft \\
\hline Wiley - Manuscript type: & Research Article \\
\hline $\begin{array}{r}\text { Date Submitted by the } \\
\text { Author: }\end{array}$ & $\mathrm{n} / \mathrm{a}$ \\
\hline Complete List of Authors: & $\begin{array}{l}\text { Tall, Issa; Southern Illinois University Carbondale, Mathematics } \\
\text { Respondek, Witold; Institut national des Sciences Appliquees de } \\
\text { Rouen, LMI }\end{array}$ \\
\hline Keywords: & $\begin{array}{l}\text { Normal Forms, Convergence, Symmetries, Feedback } \\
\text { Transformation, Strict Feedforward }\end{array}$ \\
\hline
\end{tabular}

\section{scholaroNE \\ Manuscript Central}




\title{
Analytic Normal Forms and Symmetries of Strict Feedforward Control Systems
}

\author{
Issa Amadou Tall*and Witold Respondek \\ Southern Illinois University carbondale, Department of mathematics, \\ MC 4408, Carbondale, IL 62901, itall@math.siu.edu \\ Institut National des Sciences Appliquées de Rouen, LMI, Place Emile Blondel BP 08 , \\ 76131 Mont-Saint-Aignan, Rouen,France, wresp@insa-rouen.fr
}

\section{SUMMARY}

This paper deals with the problem of convergence of normal forms. We identify a $n$-dimensional subclass of control-affine systems, called special strict feedforward form, shortly (SSFF), possessing a normal form which is a smooth (resp. analytic) counterpart of the formal normal form of Kang. We provide a constructive algorithm and illustrate by several examples. The second part of the paper is concerned about symmetries of single-input control systems. We show that any symmetry of a smooth system in special strict feedforward form is conjugated to a scaling translation and any 1-parameter family of symmetries is conjugated to a family of scaling translations along the first variable. We compute explicitly those symmetries by finding the conjugating diffeomorphism. We illustrate our results by computing the symmetries of the Cart-Pole system.

Copyright (c) 2009 John Wiley \& Sons, Ltd.

KEY WORDS: Normal Forms, Feedback Transformation, Convergence, Strict Feedforward, Symmetries

\section{Introduction}

In the past twenty five years the problem of feedback equivalence of control systems under change of coordinates and input has been studied extensively. Several methods have been proposed to deal with the problem of transforming the nonlinear control system

$$
\Pi: \dot{x}=f(x, u), \quad x \in \mathbb{R}^{n}, u \in \mathbb{R}^{m}
$$

into a simpler form

$$
\tilde{\Pi}: \dot{z}=\tilde{f}(z, v), \quad z \in \mathbb{R}^{n}, v \in \mathbb{R}^{m}
$$

by an invertible feedback transformation of the form

$$
\begin{aligned}
\Gamma: \quad z & =\phi(x) \\
u & =\gamma(x, v)
\end{aligned}
$$

*Correspondence to: Southern Illinois University carbondale, Department of mathematics, MC 4408, Carbondale, IL 62901, itall@math.siu.edu

Copyright (c) 2009 John Wiley \& Sons, Ltd. 
where the dynamics of the equivalent system are given by

$$
\tilde{f}(z, v)=\mathrm{d} \phi\left(\phi^{-1}(z)\right) \cdot f\left(\phi^{-1}(z), \gamma\left(\phi^{-1}(z), v\right)\right) .
$$

When the system $\tilde{\Pi}$ takes its simplest form $\dot{z}=A z+B v$, that is $\tilde{\Pi}$ is linear, then the system $\Pi$ is said to be linearizable via feedback. Necessary and sufficient geometric conditions for this to be the case have been given in [16] and [19]. Except for the planar case, those conditions turn out to be restrictive and a natural problem of finding normal forms for non linearizable systems arose and has been extensively studied during the last two decades (see, e.g., [6], [7], [20], [22], [23], [24], [27], [37], [61], [62] and the recent survey [42]).

A very fruitful approach leading to normal forms has been proposed by Kang and Krener [24] and then followed by Kang [22], [23]. Their idea, which is closely related with classical Poincaré's technique for linearization of dynamical systems (see e.g. [1]), is to analyze, step by step, the action of the Taylor series expansion of the feedback transformation $\Gamma$ on the Taylor series expansion of the system $\Pi$. Using that approach, results on normal forms of single-input control systems with controllable linearization have been obtained by Kang and Krener [24] for the quadratic terms, and then generalized by Kang [22] for higher order terms. The results of Kang and Krener [24],[22] have been completed by Tall and Respondek who obtained canonical forms and dual canonical forms for single-input nonlinear control systems with controllable linearization [47], [48] and then with uncontrollable linearization [49] (see also [29]). Recently those results have been generalized by Tall [45], [46] to multi-input nonlinear control systems.

The theory of normal forms, although formal, has been very useful in analyzing control systems. Using this method, bifurcations of nonlinear systems were treated in [25], [26] and the references therein, a complete description of symmetries around equilibria were presented in [38], [39], a characterization of systems equivalent to feedforward forms in [52], [53]. Their counterparts, in the discrete case, have also been obtained using a similar approach $[9,10,11,12,13,14,15]$.

The convergence of these normal forms and their normalizing transformations in the $C^{\infty}$ and analytic categories is still an open problem (see [4]).

A starting point is a result of Kang [22] derived from [27], and [28] (see also [17]) stating that, if an analytic control system is linearizable by a formal transformation, then it is linearizable by an analytic transformation. Kang [22] also gave a class of non linearizable 3-dimensional analytic control systems which are equivalent to their normal forms by analytic transformations. In [57], we gave the largest class ever of $n$-dimensional systems, namely the subclass of special strict feedforward forms, that can be brought to their normal form via smooth and analytic feedback transformations. Notice however, that $C^{\infty}$-smooth and/or analytic normal forms were obtained in [6], [18], [20], [36], [43], [63] via singularity theory methods.

We will first address the problem of convergence of normal forms in section 3 by providing the largest class ever of smooth (resp. analytic) control systems that can be brought to their normal forms via smooth (resp. analytic) feedback transformations. The class of special strict feedforward forms we consider here (see definition later) is a generalization of that, of the same name, studied in [56] with the difference that the linearization is uncontrollable.

Although it is not clear if any smooth (resp. analytic) strict feedforward system can be brought to its smooth (resp. analytic) normal form, we will define in section 4 a strict feedforward normal form, that is close as much as possible to the normal form, to which 
any smooth (resp. analytic) strict feedforward form can be transformed via smooth (resp. analytic) feedback transformation.

For simplicity of notations, we will deal with single-input nonlinear control system of the form

$$
\Pi: \dot{x}=f(x, u),
$$

where $x \in \mathbb{R}^{n}$ and $u \in \mathbb{R}$. This system is in strict feedforward form if we have

$$
\left\{\begin{array}{rll}
\dot{x}_{1} & =f_{1}\left(x_{2}, \ldots, x_{n}, u\right) \\
& \ldots \\
\dot{x}_{n-1} & =f_{n-1}\left(x_{n}, u\right) \\
\dot{x}_{n} & =f_{n}(u) .
\end{array}\right.
$$

A basic structural property of systems in strict feedforward form is that their solutions can be found by quadratures. Indeed, knowing $u(t)$ we integrate $f_{n}(u(t))$ to get $x_{n}(t)$, then we integrate $f_{n-1}\left(x_{n}(t), u(t)\right)$ to get $x_{n-1}(t)$, we keep doing that, and finally we integrate $f_{1}\left(x_{2}(t), \ldots, x_{n}(t), u(t)\right)$ to get $x_{1}(t)$.

Another property, crucial in applications, of systems in (strict) feedforward form is that we can construct for them a stabilizing feedback. This important result goes back to Teel [59] and has been followed by a growing literature on stabilization and tracking for systems in (strict) feedforward form (see e.g. [21], [32], [44], [60], [3], [33]).

The natural question of which systems are equivalent to (strict) feedforward forms arose and has been investigated by several authors. In [31], the problem of transforming a system, affine with respect to controls, into (strict) feedforward form via a diffeomorphism, i.e., via a nonlinear change of coordinates, was studied. A geometric description of systems in feedforward form has been given in [2]. Using the formal approach, we proposed a step-by-step constructive method to bring a system into a feedforward form in [52] and strict feedforward form in [53]. Recently, (see [40]), we have shown that feedback equivalence (resp. state-space equivalence) to the strict feedforward form can be characterized by the existence of a sequence of infinitesimal symmetries (resp. strong infinitesimal symmetries) of the system.

Another topic of interest that we have been investigating is about symmetries of nonlinear systems. We showed, even in the formal case, that there is a strong connection between the existence of symmetries and the feedback equivalence to (strict) feedforward systems.

We will further that topic here by providing explicit symmetries of systems in strict feedforward form via the smooth (resp. analytic) normalizing feedback transformation constructed in section 3 .

The paper is organized as follows. In section 2 we will recall the Kang normal form and our canonical form for single-input control systems. Analytic normal forms for strict feedforward and special strict feedforward systems are given in section 3, followed by their proofs. In section 4, we discuss symmetries of control systems. Illustrative examples (cart-pole, Kapitsa pendulum, etc) are given throughout the sections.

\section{Normal and Canonical Forms}

We start by briefly reviewing the results on normal and canonical forms obtained using the formal approach. 


\subsection{Formal Normal Forms}

All objects, i.e., functions, maps, vector fields, control systems, etc., are considered in a neighborhood of $0 \in \mathbb{R}^{n}$ and assumed to be $C^{\infty}$-smooth (or real analytic, if explicitly stated). Let $h$ be a smooth function. By

$$
h(x)=h^{[0]}(x)+h^{[1]}(x)+h^{[2]}(x)+\cdots=\sum_{m=0}^{\infty} h^{[m]}(x)
$$

we denote its Taylor expansion around zero, where $h^{[\mathrm{m}]}(x)$ stands for a homogeneous polynomial of degree $m$.

Similarly, for a map $\phi$ of an open subset of $\mathbb{R}^{n}$ to $\mathbb{R}^{n}$ (resp. for a vector field $f$ on an open subset of $\mathbb{R}^{n}$ ) we will denote by $\phi^{[m]}$ (resp. by $f^{[m]}$ ) the term of degree $m$ of its Taylor expansion at zero, i.e., each component $\phi_{j}^{[m]}$ of $\phi^{[m]}$ (resp. $f_{j}^{[m]}$ of $f^{[m]}$ ) is a homogeneous polynomial of degree $m$ in $x$.

Consider the Taylor series expansion of the single-input system $\Pi$, given by

$$
\Pi^{\infty}: \dot{x}=f(x, u)=F x+G u+\sum_{m=2}^{\infty} f^{[m]}(x, u),
$$

where $F=\frac{\partial f}{\partial x}(0,0)$ and $G=\frac{\partial f}{\partial u}(0,0)$. Except otherwise stated, we will assume the linear approximation around the origin to be controllable.

Consider also the Taylor series expansion $\Gamma^{\infty}$ of the feedback transformation $\Gamma$ given by

$$
\begin{aligned}
z & =\phi(x)=T x+\sum_{m=2}^{\infty} \phi^{[m]}(x) \\
\Gamma^{\infty}: \begin{array}{l}
{[x} \\
u
\end{array} & =\gamma(x, v)=K x+L v+\sum_{m=2}^{\infty} \gamma^{[m]}(x, v),
\end{aligned}
$$

where the matrix $T$ is invertible and $L \neq 0$. The action of $\Gamma^{\infty}$ on the system $\Pi^{\infty}$ step by step leads to the following normal form obtained by Kang [22] (see also [24] and [47]).

Theorem 2.1. The control system $\Pi^{\infty}$, defined by $(2.1)$, is feedback equivalent, by a formal transformation $\Gamma^{\infty}$ of the form (2.2), to the formal normal form

$$
\Pi_{N F}^{\infty}: \dot{z}=A z+B v+\sum_{m=2}^{\infty} \bar{f}^{[m]}(z, v)
$$

where $(A, B)$ is the Brunovský canonical form and for any $m \geq 2$, we have

$$
\bar{f}_{j}^{[m]}(z, v)=\left\{\begin{array}{lc}
\sum_{i=j+2}^{n+1} z_{i}^{2} P_{j, i}^{[m-2]}\left(z_{1}, \ldots, z_{i}\right), & 1 \leq j \leq n-1, \\
0, & j=n,
\end{array}\right.
$$

with $P_{j, i}^{[m-2]}$ being homogeneous polynomials of degree $m-2$ of the indicated variables, and $z_{n+1}=v$. 
The Kang normal form has been re-normalized [47] to obtain a canonical form

$$
\Pi_{C F}^{\infty}: \dot{z}=A z+B v+\sum_{m=m_{0}}^{\infty} \bar{f}^{[m]}(z, v),
$$

with the components $\bar{f}_{j}^{[m]}(z, v)$ satisfying (2.3) and, in addition

$$
\frac{\partial^{m_{0}} \bar{f}_{j^{*}}^{\left[m_{0}\right]}}{\partial z_{1}^{i_{1}} \cdots \partial z_{n-s}^{i_{n-s}}}= \pm 1 .
$$

Moreover, for any $m \geq m_{0}+1$,

$$
\frac{\partial^{m_{0}} \bar{f}_{j^{*}}^{[m]}}{\partial z_{1}^{i_{1}} \cdots \partial z_{n-s}^{i_{n-s}}}\left(z_{1}, 0, \ldots, 0\right)=0 .
$$

For the definitions of the integers $m_{0}, j^{*}$, and $s$, we refer the reader to [47].

The importance of the canonical form resides in the fact that two systems $\Sigma_{1}^{\infty}$ and $\Sigma_{2}^{\infty}$ are formally feedback equivalent if and only if their canonical forms $\Sigma_{1, C F}^{\infty}$ and $\Sigma_{2, C F}^{\infty}$ coincide. The canonical form played a key role in computing the symmetries of control systems.

If the linearization of the system around the origin is uncontrollable, we introduced weights corresponding to the uncontrollable variables [49]. Assuming the linearly uncontrollable part to be of dimension $s$, we split the coordinates as $\left(x_{1}, \ldots, x_{s}\right)$, denoting the uncontrollable variables, and $\left(x_{s+1}, \ldots, x_{n}\right)$, denoting the controllable variables. We then proved [49] (see [55]) that any single-input system, with uncontrollable linearization, is feedback equivalent to a weighted normal form

$$
\Pi_{N F}^{\infty}:\left\{\begin{aligned}
\dot{z}_{j} & =R_{j}\left(z_{1}, \ldots, z_{s}\right)+z_{s+1} S_{j}\left(z_{1}, \ldots, z_{s}\right)+\sum_{i=s+1}^{n+1} z_{i}^{2} Q_{j, i}^{\infty}\left(z_{1}, \ldots, z_{i}\right), 1 \leq j \leq s \\
\dot{z}_{j} & =z_{j+1}+\sum_{i=j+2}^{n+1} z_{i}^{2} P_{j, i}^{\infty}\left(z_{1}, \ldots, z_{i}\right), \quad s+1 \leq j \leq n-1 \\
\dot{z}_{n} & =z_{n+1}=v,
\end{aligned}\right.
$$

where the functions $Q_{j, i}^{\infty}$ and $P_{j, i}^{\infty}$ are formal power series in the controllable variables $z_{s+1}, \ldots, z_{n}, v$ whose coefficients are smooth (resp. analytic) functions of the uncontrollable variables $\left(z_{1}, \ldots, z_{s}\right)$. Those results stand for the single-input case, and have been generalized in the multi-input case $[45,46]$.

The problem whether an analogous result holds in the smooth (resp. analytic) category is actually a challenging question, which can be formulated as whether for a smooth (resp. analytic) system $\Pi$ the normalizing feedback transformation $\Gamma^{\infty}$ gives rise to a smooth (resp. convergent) $\Gamma$ and thus leads to a smooth (resp. analytic) normal form $\Pi_{N F}$ or canonical form $\Pi_{C F}$. One of the difficulties resides in the fact that it is not clear at all how to express, in terms of the original system, homogeneous invariants transformed via an infinite composition of homogeneous feedback transformations. We will study in this paper a special class of smooth (resp. analytic) control systems, namely special strict feedforward systems, that can be brought to their thus normal form by smooth (resp. analytic) transformations. 
3. Smooth and Analytic Normal Forms

Let start by recalling the results obtained by Kang for 3-dimensional systems. He pointed out that any system of the form

$$
\left\{\begin{array}{l}
\dot{x}_{1}=x_{2}+f_{1,2}\left(x_{2}\right)+x_{3} f_{1,3}\left(x_{2}\right)+x_{3}^{2} P\left(x_{1}, x_{2}, x_{3}\right) \\
\dot{x}_{2}=x_{3}+x_{3} f_{2,3}\left(x_{2}, x_{3}\right) \\
\dot{x}_{3}=u
\end{array}\right.
$$

where $f_{1,2}, f_{1,3}$, and $f_{2,3}$ are analytic functions, is feedback equivalent to its normal form

$$
\left\{\begin{array}{l}
\dot{z}_{1}=z_{2}+z_{3}^{2} \tilde{P}\left(z_{1}, z_{2}, z_{3}\right) \\
\dot{z}_{2}=z_{3} \\
\dot{z}_{3}=v
\end{array}\right.
$$

Indeed, the change of coordinates and feedback

$$
\begin{aligned}
z_{1} & =x_{1}-\int_{0}^{x_{2}} f_{1,3}(\epsilon) \mathrm{d} \epsilon \\
z_{2} & =x_{2}+f_{1,2}\left(x_{2}\right) \\
z_{3} & =x_{3}+x_{3} f_{2,3}\left(x_{2}, x_{3}\right) \\
v & =\dot{z}_{3}=\left(\partial z_{3} / \partial x_{1}\right) \dot{x}_{1}+\left(\partial z_{3} / \partial x_{2}\right) \dot{x}_{2}+\left(\partial z_{3} / \partial x_{3}\right) \dot{x}_{3}
\end{aligned}
$$

do the job.

He also gave a class of 3-dimensional systems with one uncontrollable mode, namely, systems of the form

$$
\left\{\begin{array}{l}
\dot{x}_{0}=\lambda x_{0}+f_{0}\left(x_{0}, x_{1}, x_{2}\right) \\
\dot{x}_{1}=x_{2}+f_{1}\left(x_{0}, x_{1}, x_{2}\right) \\
\dot{x}_{2}=u
\end{array}\right.
$$

that can be brought to a normal form

$$
\left\{\begin{array}{l}
\dot{z}_{0}=\lambda z_{0}+z_{1} Q_{0}\left(z_{0}\right)+z_{1}^{2} Q_{1}\left(z_{0}, z_{1}\right)+z_{2}^{2} P\left(z_{0}, z_{1}, z_{2}\right) \\
\dot{z}_{1}=z_{2} \\
\dot{z}_{2}=v
\end{array}\right.
$$

with $Q_{0}\left(z_{0}\right) \equiv 0$ if $\lambda \neq 0$.

Notice that while the first class is linearly controllable, the second class has a controllability index $p=2$ and is the analytic counterpart of our weighted normal form when $s=1$ and $n=3$.

In the following we will give an $n$-dimensional class of smooth (resp. analytic) control systems, with uncontrollable linearization, that can be brought to their normal form (weighted normal form) via smooth (resp. analytic) feedback transformation.

Consider the class of smooth (resp. analytic) single-input control systems

$$
\Pi: \dot{x}=f(x, u),
$$

either locally in a neighborhood $X \times U$ of $(0,0) \in \mathbb{R}^{n} \times \mathbb{R}$ or globally on $\mathbb{R}^{n} \times \mathbb{R}$, in strict feedforward form (SFF), that is, such that

$$
(S F F) \quad f_{j}(x, u)=f_{j}\left(x_{j+1}, \ldots, x_{n}, u\right), \quad 1 \leq j \leq n .
$$


Notice that for any $1 \leq i \leq n$, the subsystem $\Pi^{i}$, defined as the projection of $\Pi$ onto $\mathbb{R}^{n-i+1}$ via $\pi\left(x_{1}, \ldots, x_{n}\right)=\left(x_{i}, \ldots, x_{n}\right)$, is a well defined system whose dynamics are given by

$$
\dot{x}_{j}=f_{j}\left(x_{j+1}, \ldots, x_{n}, u\right)
$$

for $i \leq j \leq n$. Define the linearizability index of the (SFF)-system to be the largest integer $p$ such that the subsystem $\Pi^{r}$, where $p+r=n$, is feedback linearizable. Clearly, the linearizability index is feedback invariant and hence the linearizability indices of two feedback equivalent systems coincide.

Notice that each component of a strict feedforward system (SFF) decomposes uniquely, locally or globally, as:

$$
\left\{\begin{array}{l}
f_{j}(x, u)=h_{j}\left(x_{j+1}\right)+F_{j}\left(x_{j+1}, \ldots, x_{n}, u\right) \\
F_{j}\left(x_{j+1}, 0, \ldots, 0\right)=0, \quad 1 \leq j \leq n \\
F_{n}=0
\end{array}\right.
$$

Let $s$ be the smallest integer such that

$$
\frac{\partial h_{j}}{\partial x_{j+1}}(0) \neq 0 \quad \text { for } s+1 \leq j \leq n,
$$

where $x_{n+1}=u$. This means, in particular, that the linearization of the system around the origin is controllable when $s=0$ and is uncontrollable when $s>0$.

A strict feedforward form for which

$$
h_{j}\left(x_{j+1}\right)=k_{j} x_{j+1}, \quad s+1 \leq j \leq r-1,
$$

for some non zero real numbers $k_{s+1}, \ldots, k_{r-1}$, will be called a special strict feedforward form, shortly, (SSFF).

The first result of this paper is stated as following.

Theorem 3.1. Consider a smooth (resp. analytic) special strict feedforward form (SSFF) given by (3.1)-(3.2)-(3.3) in a neighborhood of $(0,0) \in \mathbb{R}^{n} \times \mathbb{R}$. There exists a smooth (resp. analytic) local feedback transformation that brings the system (3.1)-(3.2)-(3.3) into the normal form

$$
\Pi_{S S F N F}:\left\{\begin{array}{rlr}
\dot{z}_{j}= & R_{j}\left(z_{j+1}, \ldots, z_{s}\right)+z_{s+1} S_{j}\left(z_{j+1}, \ldots, z_{s}\right)+\sum_{i=s+1}^{n+1} z_{i}^{2} Q_{j, i}\left(z_{j+1}, \ldots, z_{i}\right), \\
\dot{z}_{j}= & z_{j+1}+\sum_{i=j+2}^{n+1} z_{i}^{2} P_{j, i}\left(z_{j+1}, \ldots, z_{i}\right), \\
\dot{z}_{r+1}= & z_{r+2}, \\
& \ldots & \text { if } s+1 \leq j \leq r \\
\dot{z}_{n-1}= & z_{n}, \\
\dot{z}_{n} & =z_{n+1}=v,
\end{array}\right.
$$

where $R_{j}\left(z_{j+1}, \ldots, z_{s}\right)$ and $S_{j}\left(z_{j+1}, \ldots, z_{s}\right)$ are smooth (resp. analytic) functions depending on the uncontrollable variables only, $Q_{j, i}\left(z_{j+1}, \ldots, z_{i}\right)$ and $P_{j, i}\left(z_{j+1}, \ldots, z_{i}\right)$ are smooth (resp. analytic) functions of the indicated variables and $z_{n+1}=v$. Moreover, if the system is defined globally on $\mathbb{R}^{n} \times \mathbb{R}$, then so are the feedback transformation and the normal form. 
This result, although stated for strict feedforward systems, remains true even if the uncontrollable part corresponding to the variables $\left(x_{1}, \ldots, x_{s}\right)^{\top}$ is not in strict feedforward form. In other words, if the projection $\Pi^{s}$ is in strict feedforward form (with $s$ defined as above), then the system is smoothly (resp. analytically) feedback equivalent to its normal form. This provides the largest class ever of nonlinear control systems that can be brought to their normal form via a smooth (resp. analytic) feedback transformation.

When $s=0$, that is, the linearization about the origin is controllable, the normal form reduces to (see [41], [56])

$$
\Pi_{\text {SSFNF }}:\left\{\begin{array}{rll}
\dot{z}_{1} & = & z_{2}+\sum_{i=3}^{n+1} z_{i}^{2} P_{1, i}\left(z_{2}, \ldots, z_{i}\right), \\
\ldots & \\
\dot{z}_{j} & = & z_{j+1}+\sum_{i=j+2}^{n+1} z_{i}^{2} P_{j, i}\left(z_{j+1}, \ldots, z_{i}\right), \\
& \ldots & \\
\dot{z}_{r} & = & z_{r+1}+\sum_{i=r+2}^{n+1} z_{i}^{2} P_{r, i}\left(z_{r+1}, \ldots, z_{i}\right), \\
\dot{z}_{r+1} & = & z_{r+2}, \\
& \cdots & \\
\dot{z}_{n-1} & = & z_{n}, \\
\dot{z}_{n} & = & v .
\end{array}\right.
$$

A main observation is that the above normal form $\Pi_{S S F N F}$ given by (3.4) is itself a (SSFF)system and, on the other hand, it constitutes a smooth (resp. analytic) counterpart $\Pi_{N F}$ of the formal normal form $\Pi_{N F}^{\infty}$ (actually, the weighted normal form) given by Theorem 2.1. However, the convergence to the canonical form is only guaranteed in the analytic case.

A question of importance is whether we can always transform a strict feedforward form, say (3.1)-(3.2), into a special strict feedforward form (3.1)-(3.2)-(3.3). To answer that question, consider another smooth (resp. analytic) system

$$
\tilde{\Pi}: \dot{z}=\tilde{f}(z, v),
$$

in strict feedforward form (SFF), that is, such that

$$
\left\{\begin{array}{l}
\tilde{f}_{j}(z, u)=\tilde{h}_{j}\left(z_{j+1}\right)+\tilde{F}_{j}\left(z_{j+1}, \ldots, z_{n}, v\right), \\
\tilde{F}_{j}\left(z_{j+1}, 0, \ldots, 0\right)=0, \quad 1 \leq j \leq n, \\
\tilde{F}_{n}=0
\end{array}\right.
$$

Let $\tilde{q}$ denote the smallest integer such that

$$
\frac{\partial \tilde{h}_{j}}{\partial z_{j+1}}(0) \neq 0, \quad \text { for } \tilde{s}+1 \leq j \leq n .
$$

It is in the special strict feedforward form (SSFF) if

$$
\tilde{h}_{j}\left(z_{j+1}\right)=\tilde{k}_{j} z_{j+1}, \quad \tilde{s}+1 \leq j \leq \tilde{r}-1
$$

for some non zero real numbers $\tilde{k}_{\tilde{s}+1}, \ldots, \tilde{k}_{\tilde{r}-1}$. 
Theorem 3.2. If two smooth (resp. analytic) (SFF)-systems given, respectively, by (3.1)-(3.2) and (3.5)-(3.6) are feedback equivalent, then $s=\tilde{s}, r=\tilde{r}$ and

$$
\tilde{h}_{j}\left(l_{j+1} z_{j+1}\right)=l_{j} h_{j}\left(z_{j+1}\right), \quad s+1 \leq j \leq r-1,
$$

for some non zero real numbers $l_{s+1}, \ldots, l_{r-1}$.

The following corollary provides the answer to the question stated above.

Corollary 3.3. A strict feedforward system (SFF), given by (3.1)-(3.2), is feedback equivalent to the special strict feedforward form (SSFF), given by (3.5)-(3.6)-(3.7), if and only if

$$
h_{j}\left(x_{j+1}\right)=k_{j} x_{j+1},
$$

for $s+1 \leq j \leq r-1$, that is, the $\left(x_{s+1}, \ldots, x_{r-1}\right)$-part of the system is already in (SSFF) in its original coordinates.

Notice that the terms $h_{j}\left(x_{j+1}\right)$ for $s+1 \leq j \leq n$ are feedback linearizable (actually $h_{j}$ form exactly the feedback linearizable terms of the form $\Pi_{S F N F}$ given by Theorem 3.1). Basically, Theorem 3.2 and Corollary 3.3 imply that the linearizable terms $h_{j}\left(x_{j+1}\right)$, for $s+1 \leq j \leq r-1$, of a strict feedforward form (SFF) cannot be linearized (unless they are already linear) via any feedback transformation that preserves the strict feedforward structure of the system. This means that special strict feedforward forms (SSFF) define the only subclass of strict feedforward systems that can be brought to the Kang normal form $\Pi_{N F}$ still being in the strict feedforward form. Whether it is possible to bring a (SFF)-system into its normal form $\Pi_{N F}$ by a smooth (resp. analytic) transformation is unclear but if true, then the normal form $\Pi_{N F}$ will loose the structure of (SFF) (unless the system is (SSFF)). On the other hand, any strict feedforward form (SFF) can be brought to a form $\Pi_{S F N F}$, called strict feedforward normal form (introduced by the authors in [52] in the formal category), which is close as much as possible to the normal form $\Pi_{N F}$ that is generalized here as

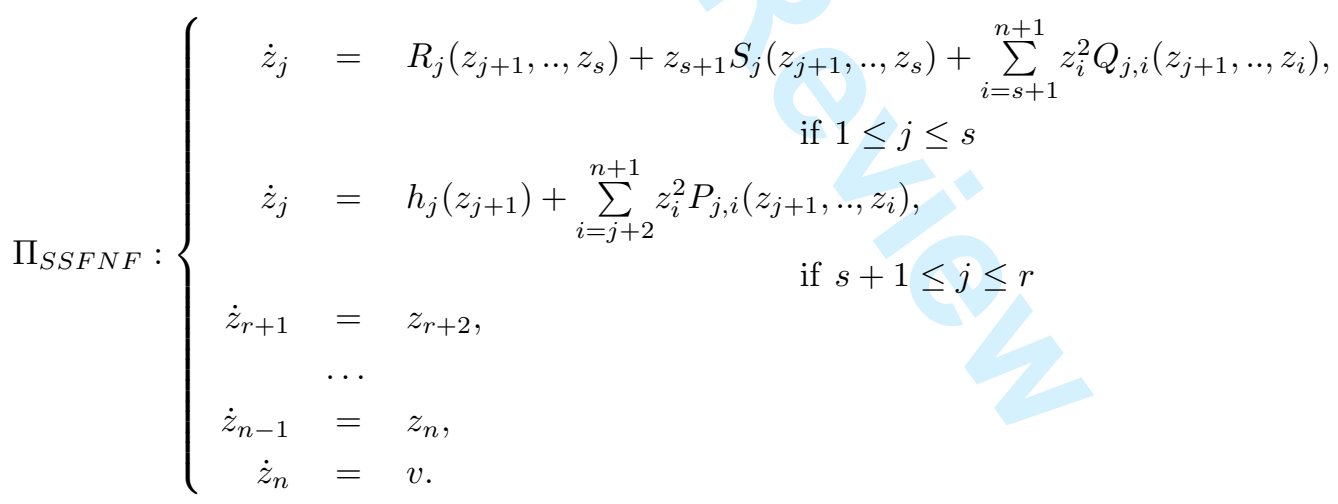

Moreover, if the system is defined globally on $\mathbb{R}^{n} \times \mathbb{R}$, then so are the feedback transformation and the normal form.

In order to explain relations between various results proved in this section, let us recall that according to Theorem 2.1, we can bring the infinite Taylor expansion $\Pi^{\infty}$ of a control system into its formal normal form $\Pi_{N F}^{\infty}$ :

$$
\Pi^{\infty} \stackrel{\text { formal }}{\longrightarrow} \Pi_{N F}^{\infty} .
$$


Theorem 3.1 says that any smooth (resp. analytic) strict feedforward system can be transformed into its strict feedforward normal forms via a smooth (resp. analytic) feedback transformation, locally or globally:

$$
\Pi_{S F F} \underset{C^{\omega}}{\stackrel{C^{\infty}}{\longrightarrow}} \Pi_{S F N F} .
$$

Provided that the linear approximation is controllable, the linearizability index of a general (SFF)-system on $\mathbb{R}^{2}$ is at least one while the linearizability index of a general control-affine system on $\mathbb{R}^{3}$ is at least two. It follows that in those two cases the functions $h_{j}$ are not invariant (compare Theorem 3.2), which implies the following:

Corollary 3.4. Any smooth (resp. analytic) strict feedforward form (SFF) on $\mathbb{R}^{2}$, given by (3.1)-(3.2), is feedback equivalent to the normal form

$$
\Pi_{S S F N F}:\left\{\begin{array}{l}
\dot{z}_{1}=z_{2}+v^{2} P_{1,3}\left(z_{2}, v\right) \\
\dot{z}_{2}=v,
\end{array}\right.
$$

where $P_{1,3}$ is a smooth (resp. analytic) function of the indicated variables.

Any smooth (resp. analytic) control-affine strict feedforward (SFF) on $\mathbb{R}^{3}$ is feedback equivalent to the normal form

$$
\Pi_{S S F N F}:\left\{\begin{aligned}
\dot{z}_{1} & =z_{2}+z_{3}^{2} P_{1,3}\left(z_{2}, z_{3}\right) \\
\dot{z}_{2} & =z_{3} \\
\dot{z}_{3} & =v .
\end{aligned}\right.
$$

where $P_{1,3}$ is a smooth (resp. analytic) function of the indicated variables.

Normal forms for strict feedforward systems on $\mathbb{R}^{2}$ with noncontrollable linearization are given in [40].

\section{Examples}

Example 3.5. Cart-Pole System. In this example we consider a cart-pole system that is represented by a cart with an inverted pendulum on it [34], [58]. The Lagrangian equations of motion for the cart-pole system are

$$
\begin{aligned}
\left(m_{1}+m_{2}\right) \ddot{q}_{1}+m_{2} l \cos \left(q_{2}\right) \ddot{q}_{2} & =m_{2} l \sin \left(q_{2}\right) \dot{q}_{2}^{2}+F \\
\cos \left(q_{2}\right) \ddot{q}_{1}+l \ddot{q}_{2} & =g \sin \left(q_{2}\right),
\end{aligned}
$$

where $m_{1}$ and $q_{1}$ are the mass and position of the cart, $m_{2}, l, q_{2} \in(-\pi / 2, \pi / 2)$ are the mass, length of the link, and angle of the pole, respectively.

Taking $\ddot{q}_{2}=u$ and applying the feedback law (see [34])

$$
F=-u l\left(m_{1}+m_{2} \sin ^{2}\left(q_{2}\right)\right) / \cos \left(q_{2}+\left(m_{1}+m_{2}\right) g \tan \left(q_{2}\right)-m_{2} l \sin \left(q_{2}\right) \dot{q}_{2}^{2},\right.
$$

the dynamics of the cart-pole system are transformed into

$$
\left\{\begin{array}{l}
\dot{x}_{1}=x_{2} \\
\dot{x}_{2}=g \tan \left(x_{3}\right)-l u / \cos \left(x_{3}\right), \\
\dot{x}_{3}=x_{4} \\
\dot{x}_{4}=u
\end{array}\right.
$$

Copyright (C) 2009 John Wiley \& Sons, Ltd. Prepared using rncauth.cls 
where we take $x_{1}=q_{1}, x_{2}=\dot{q}_{1}, x_{3}=q_{2}$, and $x_{4}=\dot{q}_{2}$.

This system is in strict feedforward form (SFF) with the linearizability index $p=2$. We showed in [38] that the diffeomorphism

$$
z=\sigma(x)=\left(\sigma_{1}(x), \sigma_{2}(x), \sigma_{3}(x), \sigma_{4}(x)\right)^{\top}
$$

defined by

$$
\begin{aligned}
z_{1} & =\sigma_{1}(x)=\mu x_{1}+\mu l \int_{0}^{x_{3}} \frac{\mathrm{ds}}{\cos s}, \\
z_{2} & =\sigma_{2}(x)=\mu x_{2}+\mu l \frac{x_{4}}{\cos x_{3}} \\
z_{3} & =\sigma_{3}(x)=\mu g \tan x_{3} \\
z_{4} & =\sigma_{4}(x)=\mu g \frac{x_{4}}{\cos ^{2} x_{3}}
\end{aligned}
$$

takes the system into its canonical form $\Sigma_{S F C F}$ :

$$
\left\{\begin{array}{l}
\dot{z}_{1}=z_{2} \\
\dot{z}_{2}=z_{3}+\frac{z_{3}}{\left(1+(g / l) z_{3}^{2}\right)^{3 / 2}} z_{4}^{2} \\
\dot{z}_{3}=z_{4} \\
\dot{z}_{4}=v
\end{array}\right.
$$

In the next example, we consider a case where the linearization about the origin is not controllable.

Example 3.6. (Kapitsa Pendulum) We consider in this example the Kapitsa Pendulum whose equations (see [5] and [8]) are given by

$$
\left\{\begin{aligned}
\dot{\alpha} & =p+\frac{w}{l} \sin \alpha \\
\dot{p} & =\left(g l-\frac{w^{2}}{l^{2}} \cos \alpha\right) \sin \alpha-\frac{w}{l} p \cos \alpha \\
\dot{z} & =w,
\end{aligned}\right.
$$

where $\alpha$ denotes the angle of the pendulum with the vertical axis $z, w$ the velocity of the suspension point $z, p$ is proportional to the generalized impulsion, $g$ is the gravity constant and $l$ the length of the pendulum.

Assume we control the acceleration $a=\dot{w}$. Introducing the coordinates system $\left(x_{1}, x_{2}, x_{3}, x_{4}\right)=(\alpha, p, z / l, w / l)$, we take $u=a / l$ as a control.

The system (3.9) considered around an equilibrium point $\left(\alpha_{0}, p_{0}, z_{0}, u_{0}\right)=(k \pi, 0,0,0)$, rewrites

$$
\left\{\begin{array}{l}
\dot{x}_{1}=x_{2}+x_{1} x_{4}+x_{4} P_{1}\left(x_{1}\right) \\
\dot{x}_{2}=\epsilon g_{0} x_{1}-x_{2} x_{4}+x_{2} x_{4} P_{2}\left(x_{1}\right)+x_{4}^{2} Q_{2}\left(x_{1}\right)+R_{2}\left(x_{1}\right) \\
\dot{x}_{3}=x_{4} \\
\dot{x}_{4}=u,
\end{array}\right.
$$

where $g_{0}=g / l, \epsilon= \pm 1, P_{1}, P_{2}, R_{2}$ are analytic functions whose 1 -jets at $(k \pi, 0,0,0)$ vanish and $Q_{2}$ an analytic function vanishing at $(k \pi, 0,0,0)$. Above, $\epsilon=1$ corresponds to $\alpha_{0}=2 n \pi$ and $\epsilon=-1$ to $\alpha_{0}=(2 n+1) \pi$. 
We can notice that the linearization about any equilibrium point is uncontrollable with a 2dimensional controllable part. Since the projection of the system on the controlled variables is in linear canonical form, hence in strict feedforward form, we expect the system to be brought to its normal form via analytic feedback transformation (according to Theorem 3.1).

Indeed, one can easily check that the quadratic feedback transformation

$$
\begin{aligned}
y_{1} & =x_{1}-x_{1} x_{3} \\
\Gamma^{2}: & =x_{2}+x_{2} x_{3} \\
y_{2} & =x_{3} \\
y_{4} & =x_{4}
\end{aligned}
$$

brings the system (3.10) into the system

$$
\left\{\begin{array}{l}
\dot{y}_{1}=y_{2}+y_{2} y_{3} \tilde{P}_{1}\left(y_{3}\right)+y_{4} \tilde{Q}_{1}\left(y_{1}, y_{3}\right) \\
\dot{y}_{2}=\epsilon g_{0} y_{1}+y_{1} y_{3} \tilde{P}_{2}\left(y_{1}, y_{3}\right)+y_{4} \tilde{Q}_{2}\left(y_{1}, y_{2}, y_{3}\right)+y_{4}^{2} \tilde{R}_{2}\left(y_{1}, y_{3}\right)+\tilde{S}_{2}\left(y_{1}\right) \\
\dot{y}_{3}=y_{4} \\
\dot{y}_{4}=u
\end{array}\right.
$$

where $\tilde{P}_{1}, \tilde{Q}_{1}, \tilde{P}_{2}, \tilde{Q}_{2}, \tilde{R}_{2}$ and $\tilde{S}_{2}$ are analytic functions.

Since the vector field defined in $\mathbb{R}^{3}$ by

$$
f=\tilde{Q}_{1}\left(y_{1}, y_{3}\right) \partial / \partial y_{1}+\tilde{Q}_{2}\left(y_{1}, y_{2}, y_{3}\right) \partial / \partial y_{2}+\partial / \partial y_{3}
$$

does not vanish neither at $(0,0,0)^{\top} \in \mathbb{R}^{3}$ nor at $(\pi, 0,0)^{\top} \in \mathbb{R}^{3}$, there exists an analytic transformation $z=\Phi(y)$ of the form

$$
\begin{aligned}
& z_{1}=\phi_{1}\left(y_{1}, y_{2}, y_{3}\right) \\
& z_{2}=\phi_{2}\left(y_{1}, y_{2}, y_{3}\right) \\
& z_{3}=y_{3}
\end{aligned}
$$

such that

$$
\left(\Phi_{*} f\right)(z)=\partial / \partial z_{3}
$$

This latter transformation, completed by $z_{4}=y_{4}$ and $u=v$, brings the system (3.11) into its normal form

$$
\left\{\begin{aligned}
\dot{z}_{1} & =z_{2}+R_{1}\left(z_{1}, z_{2}\right)+z_{3} \bar{P}_{1}\left(\bar{z}_{3}\right)+z_{4}^{2} Q_{1,4}\left(\bar{z}_{3}\right) \\
\dot{z}_{2} & =\epsilon g_{0} z_{1}+R_{2}\left(z_{1}, z_{2}\right)+z_{3} \bar{P}_{2}\left(\bar{z}_{3}\right)+z_{4}^{2} Q_{2,4}\left(\bar{z}_{3}\right) \\
\dot{z}_{3} & =z_{4} \\
\dot{z}_{4} & =v
\end{aligned}\right.
$$

where $\bar{z}_{3}=\left(z_{1}, z_{2}, z_{3}\right)$. Notice that

$$
\bar{P}_{j}\left(\bar{z}_{3}\right)=S_{j}\left(z_{1}, z_{2}\right)+z_{3} P_{j, 3}\left(z_{1}, z_{2}, z_{3}\right), \quad j=1,2 .
$$




\subsection{Proof of Theorem 3.1}

Consider a system $\Pi_{S F F}$ in strict feedforward form with linearizability index $p=n-r$ and uncontrollable linearization of dimension $s$. The system $\Pi_{S F F}$ is given by (3.1)-(3.2)(3.3). Since the projection $\Pi^{s+1}$ on $\mathbb{R}^{n-q}$ depends exclusively on the controllable variables $x_{s+1}, \ldots, x_{n}$ we will first show that $\Pi^{s+1}$ can be brought to its normal form. For simplicity in the notation, we will assume $s=0$. Notice that a short constructive proof was given in [41] and an alternative proof in [56] in the case of controllable linearization. For the sake of completeness we will provide a more detailed proof here that generalizes to the uncontrollable linearization. Without loss of generality we assume the system in the form

$$
\left\{\begin{aligned}
\dot{x}_{1} & =h_{1}\left(x_{2}\right)+F_{1}\left(x_{2}, \ldots, x_{n}, u\right) \\
\dot{x}_{2} & =h_{2}\left(x_{3}\right)+F_{2}\left(x_{3}, \ldots, x_{n}, u\right) \\
& \ldots \\
\dot{x}_{r} & =h_{r}\left(x_{r+1}\right)+F_{r}\left(x_{r+1}, \ldots, x_{n}, u\right) \\
\dot{x}_{r+1} & =x_{r+2} \\
& \ldots \\
\dot{x}_{n-1} & =x_{n} \\
\dot{x}_{n} & =u,
\end{aligned}\right.
$$

where $h_{j}$, and $F_{j}$ are smooth functions such that

$$
\begin{aligned}
h_{j}\left(x_{j+1}\right) & =k_{j} x_{j+1} \\
F_{j}\left(x_{j+1}, 0, \ldots, 0\right) & =0
\end{aligned}
$$

for any $1 \leq j \leq r-1$.

We will provide a constructive algorithmic proof by defining explicit changes of coordinates whose composition takes the system into its normal form. The algorithm will be divided into $n$ major steps. The first step consists of normalizing linear terms in $u$ in the first $n-1$ components. Then, in the second step we will normalize linear terms in $x_{n}$ in the first $n-2$ components, and so on. The algorithm consists of at most $(n-1)+(n-2)+\cdots+2+1=\frac{n(n-1)}{2}$ changes of coordinates. Actually, there are fewer changes of coordinates if the linearizability index $p>2$.

Applying the change of coordinates and feedback

$$
\begin{aligned}
z_{j} & =k_{1} \cdots k_{j-1} x_{j}, & & 1 \leq j \leq r \\
z_{r+1} & =k_{1} \cdots k_{r-1} h_{r}\left(x_{r+1}\right), & & \\
z_{j+1} & =\dot{z}_{j}, & & r+1 \leq j \leq n-1 \\
v & =\dot{z}_{n}, & &
\end{aligned}
$$

we can assume $h_{j}\left(x_{j+1}\right)=x_{j+1}$ for $1 \leq j \leq r$.

Step 1. Denote the system (3.12)-(3.13) by $\Pi_{n+1}$. We first decompose the component $F_{r}\left(x_{r+1}, \ldots, x_{n}, u\right)$ uniquely as

$$
F_{r}\left(x_{r+1}, \ldots, x_{n}, u\right)=\bar{F}_{r}\left(x_{r+1}, \ldots, x_{n}\right)+u \Theta_{r, n+1}\left(x_{r+1}, \ldots, x_{n}\right)+u^{2} P_{r, n+1}\left(x_{r+1}, \ldots, x_{n}, u\right)
$$

with $\bar{F}_{r}\left(x_{r+1}, 0, \ldots, 0\right)=0$. 
The diffeomorphism $z=\sigma_{r}^{n+1}(x)$ whose components are

$$
\begin{aligned}
& z_{j}=\sigma_{\boldsymbol{r} j}^{n+1}(x)=x_{j}, \text { if } j \neq r \\
& z_{r}=\sigma_{\boldsymbol{r} r}^{n+1}(x)=x_{r}-\int_{0}^{x_{n}} \Theta_{r, n+1}\left(x_{r+1}, \ldots, x_{n-1}, \epsilon\right) \mathrm{d} \epsilon,
\end{aligned}
$$

allows to normalize the linear terms in $u$ in the $r$ th component, and transforms it as

$$
\dot{z}_{r}=z_{r+1}+\hat{F}_{r}\left(z_{r+1}, \ldots, z_{n}\right)+u^{2} P_{r, n+1}\left(z_{r+1}, \ldots, z_{n}, u\right),
$$

where

$$
\hat{F}_{r}\left(z_{r+1}, \ldots, z_{n}\right)=\bar{F}_{r}\left(z_{r+1}, \ldots, z_{n}\right)-\sum_{k=r+1}^{n-1} z_{k+1} \int_{0}^{z_{n}} \frac{\partial \Theta_{r, n+1}}{\partial z_{k}}\left(z_{r+1}, \ldots, z_{n-1}, \epsilon\right) \mathrm{d} \epsilon .
$$

Notice that the inverse of $z=\sigma_{r}^{n+1}(x)$, say $x=\eta_{r}^{n+1}(z)$, is given by

$$
\begin{aligned}
& x_{j}=\eta_{\boldsymbol{r}_{j}}^{n+1}(z)=z_{j}, \text { if } j \neq r \\
& x_{r}=\eta_{\boldsymbol{r} r}^{n+1}(z)=z_{r}+\int_{0}^{z_{n}} \Theta_{r, n+1}\left(z_{r+1}, \ldots, z_{n-1}, \epsilon\right) \mathrm{d} \epsilon,
\end{aligned}
$$

Assume that the linear terms in $u$ are normalized in the $r$ th component through the $(i+1)$ st component, that is,

$$
\left\{\begin{aligned}
\dot{x}_{1} & =x_{2}+F_{1}\left(x_{2}, \ldots, x_{n}, u\right) \\
\dot{x}_{2} & =x_{3}+F_{2}\left(x_{3}, \ldots, x_{n}, u\right) \\
& \ldots \\
\dot{x}_{r} & =x_{r+1}+F_{r}\left(x_{r+1}, \ldots, x_{n}, u\right) \\
\dot{x}_{r+1} & =x_{r+2} \\
& \ldots \\
\dot{x}_{n-1} & =x_{n} \\
\dot{x}_{n} & =u
\end{aligned}\right.
$$

where $F_{j}$ are smooth (resp. analytic) functions such that

$$
F_{j}\left(x_{j+1}, 0, \ldots, 0\right)=0
$$

and moreover,

$$
F_{j}\left(x_{j+1}, \ldots, x_{n}, u\right)=\hat{F}_{j}\left(x_{j+1}, \ldots, x_{n}\right)+u^{2} P_{j, n+1}\left(x_{j+1}, \cdots, x_{n}, u\right)
$$

for any $i+1 \leq j \leq r$.

Decompose the $i$ th component $F_{i}\left(x_{i+1}, \ldots, x_{n}, u\right)$ uniquely as

$$
F_{i}\left(x_{i+1}, \ldots, x_{n}, u\right)=\bar{F}_{i}\left(x_{i+1}, \ldots, x_{n}\right)+u \Theta_{i, n+1}\left(x_{i+1}, \ldots, x_{n}\right)+u^{2} P_{i, n+1}\left(x_{i+1}, \ldots, x_{n}, u\right)
$$

with $\bar{F}_{i}\left(x_{i+1}, 0, \ldots, 0\right)=0$.

The diffeomorphism $z=\sigma_{i}^{n+1}(x)$ whose components are

$$
\begin{aligned}
z_{j} & =\sigma_{i j}^{n+1}(x)=x_{j}, \text { if } j \neq i \\
z_{i} & =\sigma_{i i}^{n+1}(x)=x_{i}-\int_{0}^{x_{n}} \Theta_{i, n+1}\left(x_{i+1}, \ldots, x_{n-1}, \epsilon\right) \mathrm{d} \epsilon
\end{aligned}
$$

Copyright (C) 2009 John Wiley \& Sons, Ltd.

Int. J. Robust Nonlinear Control 2009; 00:1-31 Prepared using rncauth.cls 
allows to normalize the linear terms in $u$ in the $i$ th component, and transforms that component as

$$
\dot{z}_{i}=z_{i+1}+\hat{F}_{i}\left(z_{i+1}, \ldots, z_{n}\right)+u^{2} P_{i, n+1}\left(z_{i+1}, \ldots, z_{n}, u\right)
$$

where

$$
\hat{F}_{i}\left(z_{i+1}, \ldots, z_{n}\right)=\bar{F}_{i}\left(z_{i+1}, \ldots, z_{n}\right)-\sum_{k=i+1}^{n-1} z_{k+1} \int_{0}^{z_{n}} \frac{\partial \Theta_{i, n+1}}{\partial z_{k}}\left(z_{i+1}, \ldots, z_{n-1}, \epsilon\right) \mathrm{d} \epsilon .
$$

Notice that the inverse of $z=\sigma_{i}^{n+1}(x)$, say $x=\eta_{i}^{n+1}(z)$, is given by

$$
\begin{aligned}
& x_{j}=\eta_{\boldsymbol{i} j}^{n+1}(z)=z_{j}, \text { if } j \neq i \\
& x_{i}=\eta_{\boldsymbol{i} i}^{n+1}(z)=z_{i}+\int_{0}^{z_{n}} \Theta_{i, n+1}\left(z_{i+1}, \ldots, z_{n-1}, \epsilon\right) \mathrm{d} \epsilon .
\end{aligned}
$$

The recursive method can be then applied to define diffeomorphisms $\sigma_{\boldsymbol{r}}^{n+1}, \ldots, \sigma_{\mathbf{1}}^{n+1}$, allowing to normalize the linear terms in $u$ of the corresponding component.

The composition $\sigma^{n+1}=\sigma_{1}^{n+1} \circ \cdots \circ \sigma_{r}^{n+1}$ of the successive coordinates changes transforms the system into (we reset the variable to $x$ )

$$
\left\{\begin{aligned}
\dot{x}_{1} & =x_{2}+F_{1}\left(x_{2}, \ldots, x_{n}, u\right) \\
\dot{x}_{2} & =x_{3}+F_{2}\left(x_{3}, \ldots, x_{n}, u\right) \\
& \ldots \\
\dot{x}_{r} & =x_{r+1}+F_{r}\left(x_{r+1}, \ldots, x_{n}, u\right) \\
\dot{x}_{r+1} & =x_{r+2} \\
& \ldots \\
\dot{x}_{n-1} & =x_{n} \\
\dot{x}_{n} & =u,
\end{aligned}\right.
$$

where $F_{j}$ are smooth (resp. analytic) functions such that

$$
F_{j}\left(x_{j+1}, 0, \ldots, 0\right)=0,1 \leq j \leq r
$$

and moreover,

$$
F_{j}\left(x_{j+1}, \ldots, x_{n}, u\right)=\hat{F}_{j}\left(x_{j+1}, \ldots, x_{n}\right)+u^{2} P_{j, n+1}\left(x_{j+1}, \ldots, x_{n}, u\right)
$$

for any $1 \leq j \leq r$.

Step 2. Let $\Pi_{n}=\sigma^{n+1}\left(\Pi_{n+1}\right)$ be the system $\Pi_{n+1}$ transformed via the diffeomorphism $\sigma^{n+1}$.

We deduce from above that $\hat{F}_{j}\left(x_{j+1}, 0, \ldots, 0\right)=0$ for $1 \leq j \leq r$. Let decompose the component $\hat{F}_{r}\left(x_{r+1}, \ldots, x_{n}\right)$ uniquely as following

$$
\hat{F}_{r}\left(x_{r+1}, \ldots, x_{n}\right)=\bar{F}_{r}\left(x_{r+1}, \ldots, x_{n-1}\right)+x_{n} \Theta_{r, n}\left(x_{r+1}, \ldots, x_{n-1}\right)+x_{n}^{2} P_{r, n}\left(x_{r+1}, \ldots, x_{n}\right)
$$

with $\bar{F}_{r}\left(x_{r+1}, 0, \ldots, 0\right)=0$.

The diffeomorphism $z=\sigma_{\boldsymbol{r}}^{n}(x)$ whose components are

$$
\begin{aligned}
& z_{j}=\sigma_{\boldsymbol{r} j}^{n}(x)=x_{j}, \text { if } j \neq r \\
& z_{r}=\sigma_{\boldsymbol{r} r}^{n}(x)=x_{r}-\int_{0}^{x_{n-1}} \Theta_{r, n}\left(x_{r+1}, \ldots, x_{n-2}, \epsilon\right) \mathrm{d} \epsilon,
\end{aligned}
$$

Copyright (c) 2009 John Wiley \& Sons, Ltd. Int. J. Robust Nonlinear Control 2009; 00:1-31 Prepared using rncauth.cls 
allows to normalize the linear terms in $x_{n}$ in the $r$ th component, and transforms the component as

$$
\dot{z}_{r}=z_{r+1}+\hat{F}_{r}\left(z_{r+1}, \ldots, z_{n-1}\right)+z_{n}^{2} P_{r, n}\left(z_{r+1}, \ldots, z_{n}\right)+u^{2} P_{r, n+1}\left(z_{r+1}, \ldots, z_{n}, u\right),
$$

where

$$
\hat{F}_{r}\left(z_{r+1}, \ldots, z_{n-1}\right)=\bar{F}_{r}\left(z_{r+1}, \ldots, z_{n-1}\right)-\sum_{k=r+1}^{n-2} z_{k+1} \int_{0}^{z_{n-1}} \frac{\partial \Theta_{r, n}}{\partial z_{k}}\left(z_{r+1}, \ldots, z_{n-2}, \epsilon\right) \mathrm{d} \epsilon .
$$

Following the same line as in step 1 , we would suppose that coordinates changes $\sigma_{\boldsymbol{r}}^{n}, \ldots, \sigma_{\boldsymbol{i}+\mathbf{1}}^{n}$ has been defined such that their composition transforms the original system into (we keep the variable as $x$ )

$$
\left\{\begin{aligned}
\dot{x}_{1} & =x_{2}+F_{1}\left(x_{2}, \ldots, x_{n}, u\right) \\
\dot{x}_{2} & =x_{3}+F_{2}\left(x_{3}, \ldots, x_{n}, u\right) \\
& \ldots \\
\dot{x}_{r} & =x_{r+1}+F_{r}\left(x_{r+1}, \ldots, x_{n}, u\right) \\
\dot{x}_{r+1} & =x_{r+2} \\
& \ldots \\
\dot{x}_{n-1} & =x_{n} \\
\dot{x}_{n} & =u
\end{aligned}\right.
$$

where $F_{j}$ are smooth (resp. analytic) functions such that

$$
F_{j}\left(x_{j+1}, 0, \ldots, 0\right)=0, \quad 1 \leq j \leq r
$$

and moreover,

$$
F_{j}\left(z_{j+1}, \ldots, z_{n}\right)=\hat{F}_{j}\left(x_{j+1}, \ldots, x_{n-1}\right)+x_{n}^{2} P_{j, n}\left(x_{j+1}, \ldots, x_{n}\right)+u^{2} P_{j, n+1}\left(x_{j+1}, \ldots, x_{n}, u\right)
$$

for any $i+1 \leq j \leq r$.

Decompose the $i$ th component $\hat{F}_{i}\left(x_{i+1}, \ldots, x_{n}, u\right)$ as

$$
\begin{aligned}
\hat{F}_{i}\left(x_{i+1}, \ldots, x_{n}\right)= & \bar{F}_{i}\left(x_{i+1}, \ldots, x_{n-1}\right)+x_{n} \Theta_{i, n}\left(x_{i+1}, \ldots, x_{n-1}\right) \\
& +x_{n}^{2} P_{i, n}\left(x_{i+1}, \ldots, x_{n}\right)+u^{2} P_{i, n+1}\left(x_{i+1}, \ldots, x_{n}, u\right)
\end{aligned}
$$

with $\bar{F}_{i}\left(x_{i+1}, 0, \ldots, 0\right)=0$.

The diffeomorphism $z=\sigma_{\boldsymbol{i}}^{n}(x)$ whose components are

$$
\begin{aligned}
z_{j} & =\sigma_{\boldsymbol{i} j}^{n}(x)=x_{j}, \text { if } j \neq i \\
z_{i} & =\sigma_{\boldsymbol{i} i}^{n}(x)=x_{i}-\int_{0}^{x_{n-1}} \Theta_{i, n+1}\left(x_{i+1}, \ldots, x_{n-2}, \epsilon\right) \mathrm{d} \epsilon,
\end{aligned}
$$

allows to normalize the linear terms in $x_{n}$ in the $i$ th component, and transforms the $i$ th component as

$$
\dot{z}_{i}=z_{i+1}+\hat{F}_{i}\left(z_{i+1}, \ldots, z_{n-1}\right)+z_{n}^{2} P_{i, n}\left(z_{i+1}, \ldots, z_{n}\right)+u^{2} P_{i, n+1}\left(z_{i+1}, \ldots, z_{n}, u\right),
$$


where

$$
\hat{F}_{i}\left(z_{i+1}, \ldots, z_{n-1}\right)=\bar{F}_{i}\left(z_{i+1}, \ldots, z_{n-1}\right)-\sum_{k=i+1}^{n-2} z_{k+1} \int_{0}^{z_{n-1}} \frac{\partial \Theta_{i, n}}{\partial z_{k}}\left(z_{i+1}, \ldots, z_{n-2}, \epsilon\right) \mathrm{d} \epsilon .
$$

Keeping up with the algorithm we define, successively, $\sigma_{r}^{n}, \ldots, \sigma_{1}^{n}$ whose composition is the diffeomorphism $\sigma^{n}=\sigma_{1}^{n} \circ \cdots \circ \sigma_{r}^{n}$ that takes the system into

$$
\left\{\begin{aligned}
\dot{x}_{1} & =x_{2}+F_{1}\left(x_{2}, \ldots, x_{n}, u\right) \\
\dot{x}_{2} & =x_{3}+F_{2}\left(x_{3}, \ldots, x_{n}, u\right) \\
& \ldots \\
\dot{x}_{r} & =x_{r+1}+F_{r}\left(x_{r+1}, \ldots, x_{n}, u\right) \\
\dot{x}_{r+1} & =x_{r+2} \\
& \ldots \\
\dot{x}_{n-1} & =x_{n} \\
\dot{x}_{n} & =u,
\end{aligned}\right.
$$

where $F_{j}$ are smooth (resp. analytic) functions such that

$$
F_{j}\left(z_{j+1}, \ldots, z_{n}\right)=\hat{F}_{j}\left(x_{j+1}, \ldots, x_{n-1}\right)+x_{n}^{2} P_{j, n}\left(x_{j+1}, \ldots, x_{n}\right)+u^{2} P_{j, n+1}\left(x_{j+1}, \ldots, x_{n}, u\right)
$$

for any $1 \leq j \leq r$ with

$$
\hat{F}_{j}\left(x_{j+1}, 0, \ldots, 0\right)=0 .
$$

We notice that all coordinate changes defined in step 2 depend only on the variables $x_{1}, \ldots, x_{n-1}$ but not on the variables $\left(x_{n}, u\right)$, which is the reason why the linear terms in $u$ are not created after the completion of step 1. Now, step 2, like the remaining steps of the algorithm, could have been viewed as step 1 carried over on lower dimensional systems. Indeed, taking $\hat{\Pi}_{n}$ as the restriction of system $\Pi_{n}$ on $\mathbb{R}^{n-1}$ with coordinates $\left(x_{1}, \ldots, x_{n-1}\right)$ and control $x_{n}$, step 1 would be applied to normalize the linear terms in the new control $x_{n}$.

Starting from the original system $\Pi_{n+1}$, we then define a successive sequence of diffeomorphisms $\sigma^{k+1}$ given in each step as $\sigma^{k+1}=\sigma_{\mathbf{1}}^{k+1} \circ \cdots \circ \sigma_{\boldsymbol{r}}^{k+1}$ for $k=n, n-1, \ldots, 2$ yielding a successive sequence of strict feedforward systems $\Pi_{n}, \Pi_{n-1}, \ldots, \Pi_{2}$, where for any $2 \leq k \leq n$, the system $\Pi_{k}$ is the transform of $\Pi_{k+1}$ via $\sigma^{k+1}$. Moreover, each system $\Pi_{k}$ is in the form

$$
\left\{\begin{aligned}
\dot{x}_{1} & =x_{2}+F_{1}\left(x_{2}, \ldots, x_{n}, u\right) \\
\dot{x}_{2} & =x_{3}+F_{2}\left(x_{3}, \ldots, x_{n}, u\right) \\
& \ldots \\
\dot{x}_{r} & =x_{r+1}+F_{r}\left(x_{r+1}, \ldots, x_{n}, u\right) \\
\dot{x}_{r+1} & =x_{r+2} \\
& \ldots \\
\dot{x}_{n-1} & =x_{n} \\
\dot{x}_{n} & =u,
\end{aligned}\right.
$$

where for any $1 \leq j \leq r$

$$
F_{j}\left(x_{j+1}, \ldots, x_{n}, u\right)=\hat{F}_{j}\left(x_{j+1}, \ldots, x_{k}\right)+\sum_{i=k+1}^{n+1} x_{i}^{2} P_{j, i}\left(x_{j+1}, \ldots, x_{i}\right)
$$


with $\hat{F}_{j}\left(x_{j+1}, 0, \ldots, 0\right)=0$. The functions $P_{j, i}\left(x_{j+1}, \ldots, x_{i}\right)$ are smooth (resp. analytic) in their arguments, and are zero if $i \leq j+1$.

The composition $\sigma(x)=\sigma^{3} \circ \cdots \circ \sigma^{n+1}(x)$ of these diffeomorphisms transforms the original system $\Pi_{n+1}$ into its strict feedforward normal form, which indeed coincides with $\Pi_{2}$.

To complete the proof we need to show that the uncontrollable part can be brought to a normal form without changing the components of the controllable part, already in normal form.

Reconsider $\Pi_{S F F}$ given by (3.1)-(3.2)-(3.3), and assume its linear uncontrollable part to be of dimension $s$. We will denote, for the sake of clarity, the controllable variables $x_{s+1}, \ldots, x_{n}$ by $\boldsymbol{x}_{1}, \ldots, \boldsymbol{x}_{m}$, where $m=n-s$. Without loss of generality, we can assume $\Pi_{S F F}$ in the form

$$
\Pi_{S F F}:\left\{\begin{array}{rll}
\dot{x}_{1} & = & F_{1}\left(x_{2}, \ldots, x_{s}, \boldsymbol{x}_{1}, \ldots, \boldsymbol{x}_{m}, u\right), \\
\dot{x}_{j} & = & F_{j}\left(x_{j+1}, \ldots, x_{s}, \boldsymbol{x}_{1}, \ldots, \boldsymbol{x}_{m}, u\right), \\
\dot{x}_{s} & = & F_{s}\left(\boldsymbol{x}_{1}, \ldots, \boldsymbol{x}_{m}, u\right), \\
\dot{\boldsymbol{x}}_{1} & = & \boldsymbol{x}_{2}+\sum_{i=3}^{n+1} \boldsymbol{x}_{i}^{2} P_{1, i}\left(\boldsymbol{x}_{2}, \ldots, \boldsymbol{x}_{i}\right) \\
& \ldots & \\
\dot{\boldsymbol{x}}_{j} & = & \boldsymbol{x}_{j+1}+\sum_{i=j+2}^{n+1} \boldsymbol{x}_{i}^{2} P_{j, i}\left(\boldsymbol{x}_{j+1}, \ldots, \boldsymbol{x}_{i}\right) \\
& \ldots \\
\dot{\boldsymbol{x}}_{m-1} & =\boldsymbol{x}_{m}+u^{2} P_{m-1, m+1}\left(\boldsymbol{x}_{m}, u\right) \\
\dot{\boldsymbol{x}}_{m} & =u .
\end{array}\right.
$$

The projection $\Pi^{s+1}$ is already in normal form following the normalization algorithm underlined above.

This part of the proof follows a similar line as previously. Decompose the component $F_{s}\left(\boldsymbol{x}_{1}, \ldots, \boldsymbol{x}_{m}, u\right)$, uniquely as:

$$
F_{s}\left(\boldsymbol{x}_{1}, \ldots, \boldsymbol{x}_{m}, u\right)=\hat{F}_{s}\left(\boldsymbol{x}_{1}, \ldots, \boldsymbol{x}_{m}\right)+u Q_{s}\left(\boldsymbol{x}_{1}, \ldots, \boldsymbol{x}_{m}\right)+u^{2} P_{s}\left(\boldsymbol{x}_{1}, \ldots, \boldsymbol{x}_{m}, u\right) .
$$

The change of coordinates $(z, \boldsymbol{x})=\left(\tau_{\boldsymbol{s}}^{m+1}(x), \boldsymbol{x}\right)$ whose components are defined by

$$
\begin{aligned}
& z_{j}=\tau_{\boldsymbol{s} j}^{m+1}(x)=x_{j}, \quad 1 \leq j \leq s-1, \\
& z_{s}=\tau_{\boldsymbol{s} s}^{m+1}(x)=x_{s}-\int_{0}^{\boldsymbol{x}_{m}} Q_{s}\left(\boldsymbol{x}_{1}, . ., \boldsymbol{x}_{m-1}, \epsilon\right) \mathrm{d} \epsilon
\end{aligned}
$$

allows to cancel the linear terms in $u$ in the $s$ th component. Assume that linear terms in $u$ in the $s$ th-component through the $(i+1)$ st component have been canceled. Then decompose the $i$ th component $F_{i}\left(x_{i+1}, \ldots, x_{s}, \boldsymbol{x}_{1}, \ldots, \boldsymbol{x}_{m}, u\right)$ uniquely as follows:

$$
\begin{aligned}
F_{i}\left(x_{i+1}, \ldots, x_{s}, \boldsymbol{x}_{1}, \ldots, \boldsymbol{x}_{m}, u\right)= & \hat{F}_{i}\left(x_{i+1}, \ldots, x_{s}, \boldsymbol{x}_{1}, \ldots, \boldsymbol{x}_{m}\right)+u Q_{i}\left(x_{i+1}, \ldots, x_{s}, \boldsymbol{x}_{1}, \ldots, \boldsymbol{x}_{m}\right) \\
& +u^{2} P_{i}\left(x_{i+1}, \ldots, x_{s}, \boldsymbol{x}_{1}, \ldots, \boldsymbol{x}_{m}, u\right) .
\end{aligned}
$$

Copyright (c) 2009 John Wiley \& Sons, Ltd.

Int. J. Robust Nonlinear Control 2009; 00:1-31

Prepared using rncauth.cls 
The change of coordinates $(z, \boldsymbol{x})=\left(\tau_{\boldsymbol{i}}^{m+1}(x), \boldsymbol{x}\right)$ whose first $s$ components are defined by

$$
\begin{aligned}
z_{j} & =\tau_{\boldsymbol{i} j}^{m+1}(x)=x_{j}, \quad 1 \leq j \leq i-1, \\
z_{i} & =\tau_{\boldsymbol{i} i}^{m+1}(x)=x_{i}-\int_{0}^{\boldsymbol{x}_{m}} Q_{i}\left(x_{i+1}, . ., x_{s}, \boldsymbol{x}_{1}, . ., \boldsymbol{x}_{m-1}, \epsilon\right) \mathrm{d} \epsilon \\
z_{j} & =\tau_{\boldsymbol{i} j}^{m+1}(x)=x_{j}, \quad i+1 \leq j \leq s
\end{aligned}
$$

allows to cancel the linear terms in $u$ in the $i$ th component.

The composition $\tau^{m+1}=\tau_{1}^{m+1} \circ \cdots \circ \tau_{s}^{m+1}$ allows to cancel all linear terms in $u$ in all $s$ components.

Similarly, we define transformations $\tau^{m}, \ldots, \tau^{2}$, where for any $2 \leq k \leq m+1, \tau^{k}=\tau_{1}^{k} \circ \cdots \circ \tau_{\boldsymbol{s}}^{k}$ is the transformation that linearizes the terms in $\boldsymbol{x}_{k}$ in all the $s$ components. Notice that the linear terms in $\boldsymbol{x}_{1}$ cannot be canceled in any of the first $s$ components. The composition $\tau=\tau^{2} \circ \cdots \circ \tau^{m+1}$ takes the system into its normal form. Each transformation is smooth (resp. analytic) and the algorithm involves a finite number of such transformations whose inverses are also smooth (resp. analytic). Moreover, due to the structure of the strict feedforward form, the components of the controllable part remain unchanged during the normalization of the uncontrollable part. This completes the proof of the theorem.

\section{Symmetries of Nonlinear Systems}

We will first recall our results on symmetries obtained in the single-input case using the canonical form. In the second subsection, we will give explicit symmetries of strict feedforward systems, and finally we will discuss, in term of symmetries, the feedback equivalence to a strict feedforward system

\subsection{Symmetries via Canonical Form}

Consider the single-input control-affine system

$$
\Sigma: \dot{x}=f(x)+g(x) u,
$$

where $x \in X$, is an open subset of $\mathbb{R}^{n}$, and $u \in U=\mathbb{R}, f$ and $g$ are smooth vector fields on $X$. The field of admissible velocities is the field of affine lines

$$
\mathcal{A}(x)=\{f(x)+u g(x): u \in \mathbb{R}\} \subset T_{x} X .
$$

A diffeomorphism $\psi: X \longrightarrow X$ is a symmetry of $\Sigma$ if it preserves the field of affine lines $\mathcal{A}$ (in other words, the affine distribution $\mathcal{A}$ of rank 1), that is, if $\psi_{*} \mathcal{A}=\mathcal{A}$.

A local symmetry at $p \in X$ is a local diffeomorphism $\psi$ of $X_{0}$ onto $X_{1}$, where $X_{0}$ and $X_{1}$ are, respectively, neighborhoods of $p$ and $\psi(p)$, such that

$$
\left(\psi_{*} \mathcal{A}\right)(q)=\mathcal{A}(q) \text { for any } q \in X_{1} .
$$

A local symmetry $\psi$ at $p$ is called a stationary symmetry if $\psi(p)=p$ and a nonstationary symmetry if $\psi(p) \neq p$.

Symmetries take a very simple form if we bring the system into its canonical form. Indeed, we have the following result (see [38] and [39] for proofs and details): 
Proposition 4.1. Assume that the system $\Sigma$ is analytic, the linear approximation $(F, G)$ of $\Sigma$ at an equilibrium point $p$ is controllable and $\Sigma$ is not locally feedback linearizable at $p$. Assume, moreover, that the local feedback transformation, bringing $\Sigma$ into its canonical form $\Sigma_{C F}$, is analytic at $p$.

(i) $\Sigma$ admits a nontrivial local stationary symmetry if and only if the drift $\bar{f}(x)=A x+\sum_{m=m_{0}}^{\infty} \bar{f}^{[m]}(x)$ of the canonical form $\Sigma_{C F}^{\infty}$ satisfies

$$
\bar{f}(x)=-\bar{f}(-x),
$$

that is, the system is odd.

(ii) $\Sigma$ admits a nontrivial local nonstationary symmetry if and only if the drift $\bar{f}(x)$ of the canonical form $\Sigma_{C F}^{\infty}$ satisfies

$$
\bar{f}(x)=\bar{f}\left(x_{1}+c_{1}, x_{2}, \ldots, x_{n}\right), \quad \text { for some } c_{1} \in \mathbb{R}
$$

that is $\bar{f}$ is periodic with respect to $x_{1}$.

(iii) $\Sigma$ admits a nontrivial local 1-parameter family of symmetries if and only if the drift $\bar{f}(x)$ of the canonical form $\Sigma_{C F}^{\infty}$ satisfies

$$
\bar{f}(x)=\bar{f}\left(x_{2}, \ldots, x_{n}\right) .
$$

In the case the diffeomorphism transforming the system $\Sigma$ into its canonical form $\Sigma_{C F}$ is not analytic, we have obtained formal symmetries (see [39, 38]).

\subsection{Explicit Symmetries of Strict Feedforward Systems}

In this subsection we consider the case when $\Pi$ is affine in control, that is, the class of smooth (resp. analytic) single-input control systems in strict feedforward form (SFF)

$$
\Sigma_{S F F}:\left\{\begin{array}{l}
\dot{x}=f(x)+g(x) u, \\
f_{j}(x)=f_{j}\left(x_{j+1}, \ldots, x_{n}\right), \quad 1 \leq j \leq n-1, \\
g_{j}(x)=g_{j}\left(x_{j+1}, \ldots, x_{n}\right), \quad 1 \leq j \leq n-1 \\
f_{n}(x)=f_{n} \in \mathbb{R} \text { and } g_{n}(x)=g_{n} \in \mathbb{R}^{*} .
\end{array}\right.
$$

Following section 3 , for any $1 \leq i \leq n$, the subsystem $\Sigma_{S F F}^{i}$ denotes the projection of $\Sigma_{S F F}$ onto $\mathbb{R}^{n-i+1}$ via $\pi_{i}\left(x_{1}, \ldots, x_{n}\right)=\left(x_{i}, \ldots, x_{n}\right)$ with dynamics given by

$$
\dot{x}_{j}=f_{j}\left(x_{j+1}, \ldots, x_{n}\right)+g_{j}\left(x_{j+1}, \ldots, x_{n}\right) u, \quad i \leq j \leq n .
$$

The linearizability index of $\Sigma_{S F F}$ is thus the largest integer $p$ such that the subsystem $\Sigma_{S F F}^{r+1}$, where $p+r=n$, is feedback linearizable. We will assume here that the linear approximation around the origin is controllable which implies that $p \geq 2$. The set $\mathcal{E}=\left\{x^{e} \in \mathbb{R}^{n} \mid f\left(x^{e}\right)=0\right\}$ of equilibrium points consists of lines parallel to the $x_{1}$-axis; in other words, any equilibrium point is of the form $x^{e}=\left(c_{1}, \ldots, c_{r+1}, 0, \ldots, 0\right)^{\top}$. For any nonzero real numbers $\lambda_{1}, \ldots, \lambda_{r}, \lambda \in \mathbb{R}^{*}$ and any $c_{1}, \ldots, c_{r+1} \in \mathbb{R}$, put $\Lambda=\left(\lambda_{1}, \ldots, \lambda_{r}, \lambda, \ldots, \lambda\right)$ and $C=\left(c_{1}, \ldots, c_{r+1}, 0, \ldots, 0\right)$ and define a scaling translation by

$$
\mathbb{T}_{\Lambda, C}(x)=\left(\lambda_{1} x_{1}+c_{1}, \ldots, \lambda_{n} x_{n}+c_{n}\right)^{\top},
$$

with $c_{r+2}=\cdots=c_{n}=0$ and $\lambda_{r+1}=\cdots=\lambda_{n}=\lambda$. 
Theorem 4.2. Consider a smooth system $\Sigma_{S F F}$ in strict feedforward form with linearizability index $p=n-r$. Any symmetry $\psi$ of $\Sigma_{S F F}$ is of the form

$$
\psi=\sigma^{-1} \circ \mathbb{T}_{\Lambda, C} \circ \sigma,
$$

for a fixed $(\Lambda, C)$, where $z=\sigma(x)$ is the diffeomorphism of the transformation taking $\Sigma_{S F F}$ into its strict feedforward normal form $\Sigma_{S F N F}$ given by Definition 4.3 below. Any local 1-parameter family of symmetries $\psi_{c_{1}}$ of $\Sigma_{S F F}$ is of the same form with $c_{1} \in\left(-\epsilon_{1}, \epsilon_{1}\right)$.

Theorem 4.2 says basically that strict feedforward systems have 1-parameter families of symmetries conjugated to scaling translations. Recall that in [38] we showed that any symmetry is conjugated to at most two 1-parameter families of translations along the first variable; those translations being the only symmetries of the canonical form (Proposition 4.1).

The constant parameters $\lambda_{1}, \ldots, \lambda_{r}, \lambda$ are likely to be either +1 or -1 and will be uniquely determined by $c_{2}, \ldots, c_{r}$ (given by other equilibrium point) because, together, they should satisfy some strong conditions (SC), see below. The only free parameter is $c_{1}$. In Example 4.9 we provide a case where some of the parameters $\lambda_{1}, \ldots, \lambda_{r}, \lambda$ are not equal to +1 or -1 as well as some constants $c_{2}, \ldots, c_{r+1}$ that are non zero. We then compare the results obtained here with those of [38], and show no ambiguity between them.

The importance of this result is that we can always put a (SFF)-system into a strict feedforward normal form (SFNF) via smooth feedback transformation while the canonical form is only guaranteed in the formal category. Moreover, the feedback transformation taking the system into its strict feedforward normal form (SFNF) can be constructed explicitly, for smooth systems, see section 3.1.

The notion of strict feedforward normal form plays a crucial role in proving Theorem 4.2 and, in the affine case, takes the following form.

Definition 4.3. A smooth strict feedforward normal form, denoted $\Sigma_{S F N F}$, is a strict feedforward form

$$
\left\{\begin{aligned}
\dot{x}_{1} & =\hat{F}_{1}\left(x_{2}, \ldots, x_{n}\right) \\
& \ldots \\
\dot{x}_{r} & =\hat{F}_{r}\left(x_{r+1}, \ldots, x_{n}\right) \\
\dot{x}_{r+1} & =x_{r+2} \\
& \cdots \\
\dot{x}_{n-1} & =x_{n} \\
\dot{x}_{n} & =u
\end{aligned}\right.
$$

for which $p=n-r$ is the linearizability index and

$$
\hat{F}_{j}(x)=h_{j}\left(x_{j+1}\right)+\sum_{i=j+2}^{n} x_{i}^{2} P_{j, i}\left(x_{j+1}, \ldots, x_{i}\right)
$$

for any $1 \leq j \leq r$, where $h_{j}$ and $P_{j, i}$ are smooth functions of the indicated variables.

The above strict feedforward normal form $\Sigma_{S F N F}$ was introduced in [41], where we proved the following:

Theorem 4.4. Any smooth strict feedforward form can be transformed into a strict feedforward normal form via smooth feedback transformation. 
Remark 4.5. (i) The explicit construction of the feedback transformation (in particular, the diffeomorphism $z=\sigma(x)$ ) taking a (SFF)-system into its (SFNF), was given in the proof of Theorem 3.1. We have assume there, without loss of generality, that $h_{j}\left(x_{j+1}\right)=x_{j+1}$ but the algorithm remains the same.

Then using the commutative diagram

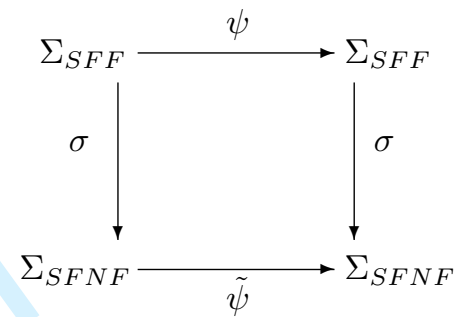

where $\tilde{\psi}$ is a symmetry of the strict feedforward normal form $\Sigma_{S F N F}$, all we will have to prove is that all $\tilde{\psi}$ 's are exhausted by scaling translations $\mathbb{T}_{\Lambda, C}$ defined above.

(ii) We will use this item to deduce, as a corollary, necessary and sufficient condition for a system to be brought to a strict feedforward form (see Theorem II.4 of [40]).

\section{B1. Proof of Theorem 4.2}

We will prove Theorem 4.2 by showing that symmetries of systems in strict feedforward normal form $\Sigma_{S F N F}$ are exhausted by scaling translations $\mathbb{T}_{\Lambda, C}$ defined above. Let us consider a system in the strict feedforward normal form $\Sigma_{S F N F}$, given by definition 4.3 .

Notice that if $\tilde{x}=\tilde{\psi}(x)$ is a symmetry of $\Sigma_{S F F}$ (in particular, of $\Sigma_{S F N F}$ ), then it preserves the structure of the strict feedforward form. Hence (see [51]), we have $\tilde{x}_{j}=\tilde{\psi}_{j}(x)=$ $\tilde{\psi}_{j}\left(x_{j}, \ldots, x_{n-1}\right)$ for $1 \leq j \leq n-1$. This implies that $\pi_{r}(\tilde{\psi})=\left(\tilde{\psi}_{r}(x), \ldots, \tilde{\psi}_{n}(x)\right)$ is a symmetry of the projection $\Sigma_{S F N F}^{r+1}$ of $\Sigma_{S F F}$ whose dynamics are

$$
\left\{\begin{aligned}
\dot{x}_{r} & =h_{r}\left(x_{r+1}\right)+\sum_{i=r+2}^{n} x_{i}^{2} P_{r, i}\left(x_{r+1}, \ldots, x_{i}\right) \\
\dot{x}_{r+1} & =x_{r+2} \\
& \ldots \\
\dot{x}_{n-1} & =x_{n} \\
\dot{x}_{n} & =u .
\end{aligned}\right.
$$

We claim that $\tilde{\psi}_{j}(x)=\tilde{\psi}_{j}\left(x_{j}\right)$ for any $r \leq j \leq n-1$. Indeed, we have $\tilde{\psi}_{n-1}(x)=$ $\tilde{\psi}_{n-1}\left(x_{n-1}\right)$. Let $k$ be the largest integer, $r \leq k \leq n-2$, such that $\frac{\partial \tilde{\psi}_{k}}{\partial x_{s}} \neq 0$ for some $s \geq k+1$ (we can take $s$ to be the largest integer that yields this property). Thus

$$
\dot{\tilde{x}}_{k}=\frac{\partial \tilde{\psi}_{k}}{\partial x_{k}} \dot{x}_{k}+\cdots+\frac{\partial \tilde{\psi}_{k}}{\partial x_{s}} x_{s+1}=\tilde{x}_{k+1}=\tilde{\psi}_{k+1}(x)
$$

gives a contradiction because $\tilde{\psi}_{k+1}(x)=\tilde{\psi}_{k+1}\left(x_{k+1}\right)$. We conclude that $\tilde{\psi}_{j}(x)=\tilde{\psi}_{j}\left(x_{j}\right)$ for $r \leq j \leq n-1$. Since

$$
\dot{\tilde{x}}_{j}=\tilde{\psi}_{j}^{\prime}\left(x_{j}\right) x_{j+1}=\tilde{x}_{j+1}=\tilde{\psi}_{j+1}\left(x_{j+1}\right),
$$


we deduce that $\tilde{\psi}_{j}\left(x_{j}\right)=\lambda_{j} x_{j}+c_{j}$ for all $r+1 \leq j \leq n-1$. Similarly we get $\tilde{\psi}_{r}\left(x_{r}\right)=\lambda_{r} x_{r}+c_{r}$ and hence

$$
\pi_{r}(\tilde{\psi}(x))=\left(\lambda_{r} x_{r}+c_{r}, \lambda_{r+1} x_{r+1}+c_{r+1}, \ldots, \lambda_{n} x_{n}+c_{n}\right)^{\top} .
$$

In fact, it is easy to see that $\lambda_{r+1}=\cdots=\lambda_{n}=\lambda$ and $c_{r+2}=\cdots=c_{n}=0$ but for homogeneity of notation, we will carry those constants as such.

Notice that $\lambda_{r}$, and the pairs $\left(\lambda_{k}, c_{k}\right), r+1 \leq k \leq n$ should satisfy the strong condition:

$$
\left.\hat{F}_{r}\left(\lambda_{r+1} x_{r+1}+c_{r+1}, \ldots, \lambda_{n} x_{n}+c_{n}\right)\right)=\lambda_{r} \hat{F}_{r}\left(x_{r+1}, \ldots, x_{n}\right),
$$

and

$$
\hat{F}_{r}\left(x_{r+1}, \ldots, x_{n}\right)=h_{r}\left(x_{r+1}\right)+\sum_{i=r+2}^{n} x_{i}^{2} P_{r, i}\left(x_{r+1}, \ldots, x_{i}\right) .
$$

We can remark that $(\mathrm{SC})_{r}$ is equivalent to the conditions

$$
h_{r}\left(\lambda_{r+1} x_{r+1}+c_{r+1}\right)=\lambda_{r} h_{r}\left(x_{r+1}\right)
$$

$$
P_{r, i}\left(\lambda_{r+1} x_{r+1}+c_{r+1}, \ldots, \lambda_{i} x_{i}+c_{i}\right)=\frac{\lambda_{r}}{\lambda_{i}^{2}} P_{r, i}\left(x_{r+1}, \ldots, x_{i}\right), r+2 \leq i \leq n .
$$

A similar argument will imply that $\tilde{\psi}_{j}(x)=\tilde{\psi}\left(x_{j}\right)$ for all $1 \leq j \leq r-1$. Taking $j=r-1$, we should have

$$
\dot{\tilde{x}}_{r-1}=\tilde{\psi}_{r-1}^{\prime}\left(x_{r-1}\right) \hat{F}_{r-1}\left(x_{r}, \ldots, x_{n}\right)=\hat{F}_{r-1}\left(\tilde{x}_{r}, \ldots, \tilde{x}_{n}\right)
$$

which implies that $\tilde{\psi}_{r-1}^{\prime}\left(x_{r-1}\right)=\lambda_{r-1}$, and consequently, $\tilde{\psi}_{r-1}\left(x_{r-1}\right)=\lambda_{r-1} x_{r-1}+c_{r-1}$.

A straightforward recurrence shows that for any $1 \leq j \leq r$, we have $\tilde{\psi}_{j}\left(x_{j}\right)=\lambda_{j} x_{j}+c_{j}$. At each step, the constant $\lambda_{j}$ is related to the pairs $\left(\lambda_{k}, c_{k}\right)$, for $j+1 \leq k \leq n$, by the strong conditions

$$
\hat{F}_{j}\left(\lambda_{j+1} x_{j+1}+c_{j+1}, \ldots, \lambda_{i} x_{n}+c_{n}\right)=\lambda_{j} \hat{F}_{j}\left(x_{j+1}, \ldots, x_{n}\right),
$$

and

$$
\hat{F}_{j}\left(x_{j+1}, \ldots, x_{n}\right)=h_{j}\left(x_{j+1}\right)+\sum_{i=j+2}^{n} x_{i}^{2} P_{j, i}\left(x_{r+1}, \ldots, x_{i}\right) .
$$

Notice that the constant $c_{1}$ can be chosen arbitrarily. The proof of Theorem 4.2 is then completed by the commutative diagram giving the explicit diffeomorphism $z=\sigma(x)$ of the feedback transformation bringing $\Sigma_{S F F}$ into its strict feedforward normal form (see Proof of Theorem 3.1).

\section{B2. Feedback Equivalence to Strict Feedforward Systems}

The problem of transforming a system, affine with respect to controls, into (strict) feedforward form via a nonlinear change of coordinates was studied in [31], and a geometric description of systems in feedforward form has been given in [2]. We proposed a step-by-step constructive method to bring a system into a feedforward form in [54] and into a strict feedforward form in [50]. 
Recently (see [40]), we have proved that feedback equivalence (resp. state-space equivalence) to the strict feedforward form can be characterized by the existence of a sequence of infinitesimal symmetries (resp. strong infinitesimal symmetries) of the system. We give here as a corollary, a restatement of the equivalence conditions obtained in [40] in terms of the symmetries of strict feedforward systems.

Corollary 4.6. Consider a smooth affine system $\Sigma$ with linearizability index $p=n-r$. The following conditions are equivalent.

(i) $\Sigma$ is, locally at $q \in X$, feedback equivalent to the affine strict feedforward form (SFF);

(ii)Each system $\Sigma_{1}, \Sigma_{2}, \ldots, \Sigma_{r}$ possesses an infinitesimal symmetry $v_{i}$, whose local flow $\gamma_{c_{i}}^{v_{i}}$ is conjugated to a scaling translation

$$
\gamma_{c_{i}}^{v_{i}}=\sigma_{i}^{-1} \circ \mathbb{T}_{\Lambda, C}^{i} \circ \sigma_{i}, \quad c_{i} \in\left(-\epsilon_{i}, \epsilon_{i}\right),
$$

where $\Sigma_{1}$ is the restriction of $\Sigma$ to a neighborhood $X_{q}$ and

$$
\Sigma_{i+1}=\Sigma_{i} / \sim_{v_{i}}, \quad 1 \leq i \leq r-1 .
$$

Above, the equivalence relation $\sim_{v_{i}}$ is induced by the local action of the 1-parameter local group $\gamma_{c_{i}}^{v_{i}}$ defined by $v_{i}$, that is, such that $q_{1} \sim_{v_{i}} q_{2}$ if and only if they belong to the same integral curve of $v_{i}$, and for any $1 \leq i \leq r-1$ the scaling translation $\mathbb{T}_{\Lambda, C}^{i}$ is the composition of $\mathbb{T}_{\Lambda, C}$ with the projection $\pi_{i}$ :

$$
\mathbb{T}_{\Lambda, C}^{i}(x)=\left(\lambda_{i} x_{i}+c_{i}, \ldots, \lambda_{r} x_{r}+c_{r}, \lambda x_{r+1}, \ldots, \lambda x_{n}\right)^{\top} .
$$

\section{Examples}

Example 4.7. (Cart-Pole Cont'd) Reconsider the cart-pole system given in Example 3.5. It has been shown that the cart-pole system can be put into its canonical form

$$
\left\{\begin{aligned}
\dot{z}_{1} & =z_{2}, \\
\dot{z}_{2} & =z_{3}+\frac{z_{3}}{\left(1+(g / l) z_{3}^{2}\right)^{3 / 2}} z_{4}^{2}, \\
\dot{z}_{3} & =z_{4} \\
\dot{z}_{4} & =v
\end{aligned}\right.
$$

via analytic feedback transformation. It is straightforward to verify that

$$
\mathbb{T}_{\mathrm{Id}, C}(z)=\left(z_{1}+c_{1}, z_{2}, z_{3}, z_{4}\right)^{\top} \quad \text { and } \quad \mathbb{T}_{-\mathrm{Id}, C}(z)=\left(-z_{1}+c_{1},-z_{2},-z_{3},-z_{4}\right)^{\top}
$$

constitute two 1-parameter families of symmetries for the canonical form. By Theorem 4 (see [38]), they exhaust all possible symmetries of the canonical form.

The symmetries of (3.8) are obtained by computing

$$
\psi(x)=\sigma^{-1} \circ \mathbb{T}_{ \pm \mathrm{Id}, C}(x) \circ \sigma(x)
$$


where the inverse $x=\eta(z)=\sigma^{-1}(z)$ is given by

$$
\begin{aligned}
x_{1} & =\eta_{1}(z)=\tilde{\mu} g z_{1}+\theta\left(z_{3}\right), \\
x_{2} & =\eta_{2}(z)=\tilde{\mu} g z_{2}-\tilde{\mu} l \frac{z_{4}}{\sqrt{1+\left(\tilde{\mu} z_{3}\right)^{2}}} \\
x_{3} & =\eta_{3}(z)=\arctan \left(\tilde{\mu} z_{3}\right), \\
x_{4} & =\eta_{4}(z)=\frac{\tilde{\mu} z_{4}}{1+\left(\tilde{\mu} z_{3}\right)^{2}}
\end{aligned}
$$

for a suitable function $\theta\left(z_{3}\right)$. It follows easily that

$$
\sigma^{-1} \circ \mathbb{T}_{\mathrm{Id}, C} \circ \sigma(x)=\mathbb{T}_{\mathrm{Id}, \bar{C}}(x) \quad \text { and } \quad \sigma^{-1} \circ \mathbb{T}_{-\mathrm{Id}, C} \circ \sigma(x)=\mathbb{T}_{-\mathrm{Id}, \bar{C}}(x)
$$

are both 1-parameter families of translations along the first component $x_{1}$ of $\left(x_{1}, x_{2}, x_{3}, x_{4}\right)^{\top}$. The meaning of the symmetries here, when expressed back in the original physical coordinates $\left(\tilde{q}_{1}, \dot{\tilde{q}}_{1}, \tilde{q}_{2}, \dot{\tilde{q}}_{2}\right)=\left(q_{1}+c_{1}, \dot{q}_{1}, q_{2}, \dot{q}_{2}\right)$, is that the experiment conducted on two similar carts traveling at the same speed (either forward or backward) yields the same conclusions.

In the following, we give examples showing that symmetries as nonstationary translations and scaling translations are possible.

Example 4.8. Consider the system in $\mathbb{R}^{4}$ described by

$$
\left\{\begin{array}{l}
\dot{x}_{1}=\sin x_{2}+x_{4}^{2} \sin x_{3} \\
\dot{x}_{2}=\sin x_{3}+x_{4}^{3} \\
\dot{x}_{3}=x_{4} \\
\dot{x}_{4}=u
\end{array}\right.
$$

This system is clearly in (SFNF) with linearizability index $p=2$. It is easy to check that the forward and backward translations

$\mathbb{T}_{\text {Id }, C}(x)=\left(x_{1}+c_{1}, x_{2}+c_{2}, x_{3}+c_{3}, x_{4}\right)^{\top} \quad$ and $\quad \mathbb{T}_{-\mathrm{Id}, C}(x)=\left(-x_{1}+c_{1},-x_{2}+c_{2},-x_{3}+c_{3},-x_{4}\right)^{\top}$ are symmetries, where $c_{2}$ and $c_{3}$ are any multiples of $2 \pi$.

Example 4.9. Consider the system

$$
\Sigma_{S F F}:\left\{\begin{aligned}
\dot{x}_{1} & =x_{2}+2 x_{2} e^{x_{3}} \sin x_{3}+2 x_{2} e^{x_{3}} x_{4}^{2}, \\
\dot{x}_{2} & =e^{x_{3}} \sin x_{3}+e^{x_{3}} x_{4}^{2}, \\
\dot{x}_{3} & =x_{4}, \\
\dot{x}_{4} & =u,
\end{aligned}\right.
$$

in strict feedforward form with linearizability index $p=2$. Due to the terms $2 x_{2} e^{x_{3}} \sin x_{3}$, this system is not in strict feedforward normal form. However, it is straightforward to check that the diffeomorphism $z=\sigma(x)$ defined by

$$
z_{1}=x_{1}-x_{2}^{2}, z_{2}=x_{2}, z_{3}=x_{3}, z_{4}=x_{4}
$$

takes $\Sigma_{S F F}$ into the strict feedforward normal form

$$
\Sigma_{S F N F}:\left\{\begin{array}{l}
\dot{z}_{1}=z_{2}, \\
\dot{z}_{2}=e^{z_{3}} \sin z_{3}+e^{z_{3}} z_{4}^{2}, \\
\dot{z}_{3}=z_{4} \\
\dot{z}_{4}=u
\end{array}\right.
$$


We can notice that the scaling translations

$$
\tilde{z}=\mathbb{T}_{\Lambda, C}(z)=\left(\lambda z_{1}+c_{1}, \lambda z_{2}, z_{3}+c_{3}, z_{4}\right)^{\top}
$$

with $c_{3}=2 k \pi, k \in \mathbb{Z}$, and $\lambda=e^{c_{3}}$ form a family of symmetries of $\Sigma_{S F N F}$ parameterized by $c_{1}$. Indeed, it is easy to see that they map $\Sigma_{S F N F}$ into $\Sigma_{S F N F}$ given, around the equilibrium $q=\left(0,0, c_{3}, 0\right)^{\top}$, by

$$
\Sigma_{S F N F}^{(q)}:\left\{\begin{array}{l}
\dot{\tilde{z}}_{1}=\tilde{z}_{2}, \\
\dot{\tilde{z}}_{2}=e^{\tilde{z}_{3}} \sin \tilde{z}_{3}+e^{\tilde{z}_{3}} \tilde{z}_{4}^{2}, \\
\dot{\tilde{z}}_{3}=\tilde{z}_{4}, \\
\dot{\tilde{z}}_{4}=u .
\end{array}\right.
$$

The composition $\tilde{x}=\sigma^{-1} \circ \mathbb{T}_{\Lambda, C} \circ \sigma(x)$ expresses the coordinates $\tilde{x}$ in terms of the coordinates $x$ as follows

$$
\begin{aligned}
& \tilde{x}_{1}=\lambda x_{1}+\left(\lambda^{2}-\lambda\right) x_{2}^{2}+c_{1}, \\
& \tilde{x}_{2}=\lambda x_{2}, \\
& \tilde{x}_{3}=x_{3}+c_{3} \\
& \tilde{x}_{4}=x_{4},
\end{aligned}
$$

where $c_{3}=2 \pi$ and $\lambda=e^{c_{3}}$.

A straightforward calculation shows that

$$
\begin{aligned}
\dot{\tilde{x}}_{1} & =\lambda\left(x_{2}+2 x_{2} e^{x_{3}} \sin x_{3}+2 x_{2} e^{x_{3}} x_{4}^{2}\right)+2\left(\lambda^{2}-\lambda\right) x_{2}\left(e^{x_{3}} \sin x_{3}+e^{x_{3}} x_{4}^{2}\right) \\
& =\lambda x_{2}+2 \lambda^{2} x_{2}\left(e^{x_{3}} \sin x_{3}+e^{x_{3}} x_{4}^{2}\right) \\
& =\tilde{x}+2 \tilde{x}_{2} e^{\tilde{x}_{3}} \sin \tilde{x}_{3}+2 \tilde{x}_{2} e^{\tilde{x}_{3}} \tilde{x}_{4}^{2}
\end{aligned}
$$

because $\lambda x_{2}=\tilde{x}_{2}$ and

$$
\lambda e^{x_{3}} \sin x_{3}=e^{x_{3}+c_{3}} \sin \left(x_{3}+c_{3}\right)=e^{\tilde{x}_{3}} \sin \tilde{x}_{3} .
$$

Similarly, we can show that

$$
\dot{\tilde{x}}_{2}=\lambda\left(e^{x_{3}} \sin x_{3}+e^{x_{3}} x_{4}^{2}\right)=e^{\tilde{x}_{3}} \sin \tilde{x}_{3}+e^{\tilde{x}_{3}} \tilde{x}_{4}^{2} .
$$

Since $\dot{\tilde{x}}_{3}=\tilde{x}_{4}$ and $\dot{\tilde{x}}_{4}=u$, it follows that the composition $\tilde{x}=\sigma^{-1} \circ \mathbb{T}_{\Lambda, C} \circ \sigma(x)$ maps $\Sigma_{S F F}$, defined around the equilibrium $(0,0,0,0)^{\top}$, into $\Sigma_{S F F}$ described, around the equilibrium $q=(0,0,2 \pi, 0)^{\top}$, by the same dynamics

$$
\Sigma_{S F F}:\left\{\begin{array}{l}
\dot{\tilde{x}}_{1}=\tilde{x}_{2}+2 \tilde{x}_{2} e^{\tilde{x}_{3}} \sin \tilde{x}_{3}+2 \tilde{x}_{2} e^{\tilde{x}_{3}} \tilde{x}_{4}^{2} \\
\dot{\tilde{x}}_{2}=e^{\tilde{x}_{3}} \sin \tilde{x}_{3}+e^{\tilde{x}_{3}} \tilde{x}_{4}^{2} \\
\dot{\tilde{x}}_{3}=\tilde{x}_{4} \\
\dot{\tilde{x}}_{4}=u
\end{array}\right.
$$


We have the commutative diagram

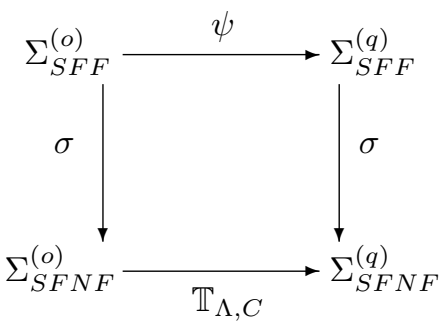

Hence $\tilde{x}=\psi(x)=\sigma^{-1} \circ \mathbb{T}_{\Lambda, C} \circ \sigma(x)$ is a 1-parameter family of symmetries of $\Sigma_{S F F}$.

For convenience of notation, we will denote $\Sigma_{S F F}$, defined around $(0,0,0,0)^{\top}$, by $\Sigma_{S F F}^{(o)}$ and the system $\Sigma_{S F F}$, defined around $q=(0,0,2 \pi, 0)^{\top}$, by $\Sigma_{S F F}^{(q)}$. The same notations apply to the systems $\Sigma_{S F N F}^{(o)}$ and $\Sigma_{S F N F}^{(q)}$.

Now, in view of the results obtained in [41], we will compute the canonical form of $\Sigma_{S F F}^{(o)}$ and the transformations taking $\Sigma_{S F F}^{(o)}$ and $\Sigma_{S F F}^{(q)}$ to this canonical form.

It is easy to verify that $y=\Phi(x)$, given by

$$
\begin{aligned}
& y_{1}=x_{1}-x_{2}^{2}, \\
& y_{2}=x_{2}, \\
& y_{3}=e^{x_{3}} \sin x_{3} \\
& y_{4}=e^{x_{3}}\left(\sin x_{3}+\cos x_{3}\right) x_{4},
\end{aligned}
$$

followed by an appropriate feedback, takes the system $\Sigma_{S F F}^{(o)}$ into its canonical form

$$
\Sigma_{S F C F}:\left\{\begin{array}{l}
\dot{y}_{1}=y_{2}, \\
\dot{y}_{2}=y_{3}+\Theta\left(y_{3}\right) y_{4}^{2}, \\
\dot{y}_{3}=y_{4}, \\
\dot{y}_{4}=v,
\end{array}\right.
$$

where $\Theta\left(y_{3}\right)=\left.\frac{1}{e^{x_{3}}\left(\sin x_{3}+\cos x_{3}\right)^{2}}\right|_{x_{3}=\theta^{-1}\left(y_{3}\right)}$ with $\theta\left(x_{3}\right)=e^{x_{3}} \sin x_{3}$.

On the other hand, applying the translation

$$
\hat{x}=T(\tilde{x})=\left(\tilde{x}_{1}, \tilde{x}_{2}, \tilde{x}_{3}-c_{3}, \tilde{x}_{4}\right)
$$

to the system $\Sigma_{S F F}^{(q)}$, we can shift back the equilibrium point to $(0,0,0,0)$. In the new coordinates, $\Sigma_{S F F}^{(q)}$ becomes

$$
\tilde{\Sigma}_{S F F}^{(o)}:\left\{\begin{array}{l}
\dot{\hat{x}}_{1}=\hat{x}_{2}+2 \lambda \hat{x}_{2}\left(e^{\hat{x}_{3}} \sin \hat{x}_{3}+e^{\hat{x}_{3}} \hat{x}_{4}^{2}\right), \\
\dot{\hat{x}}_{2}=\lambda\left(e^{\hat{x}_{3}} \sin \hat{x}_{3}+e^{\hat{x}_{3}} \hat{x}_{4}^{2}\right) \\
\dot{\hat{x}}_{3}=\hat{x}_{4} \\
\dot{\hat{x}}_{4}=u
\end{array}\right.
$$


where $\lambda=e^{c_{3}}$. The diffeomorphism $\tilde{y}=\Psi(\hat{x})$ given by

$$
\begin{aligned}
& \tilde{y}_{1}=\lambda^{-1}\left(\hat{x}_{1}-\hat{x}_{2}^{2}\right), \\
& \tilde{y}_{2}=\lambda^{-1} \hat{x}_{2}, \\
& \tilde{y}_{3}=e^{\hat{x}_{3}} \sin \hat{x}_{3} \\
& \tilde{y}_{4}=e^{\hat{x}_{3}}\left(\sin \hat{x}_{3}+\cos \hat{x}_{3}\right) \hat{x}_{4},
\end{aligned}
$$

followed by an appropriate feedback, takes the system $\tilde{\Sigma}_{S F F}^{(o)}$ into its canonical form

$$
\Sigma_{S F C F}:\left\{\begin{array}{l}
\dot{\tilde{y}}_{1}=\tilde{y}_{2}, \\
\dot{\tilde{y}}_{2}=\tilde{y}_{3}+\Theta\left(\tilde{y}_{3}\right) \tilde{y}_{4}^{2}, \\
\dot{\tilde{y}}_{3}=\tilde{y}_{4}, \\
\dot{\tilde{y}}_{4}=v .
\end{array}\right.
$$

It follows that the composition $\tilde{y}=\Psi \circ T \circ \psi \circ \Phi^{-1}(y)$ is a 1-parameter family of symmetries of the canonical form according to the diagram.

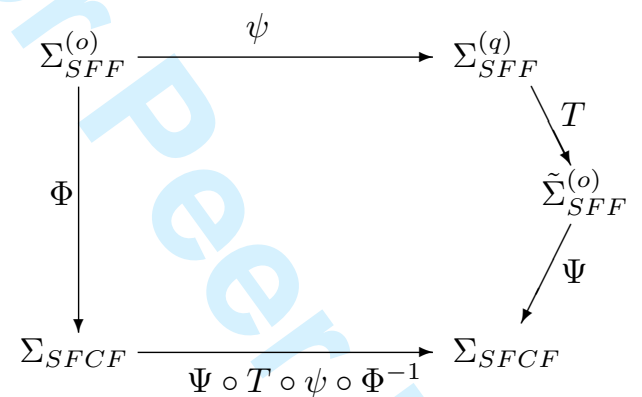

We explicitly find this family of symmetries by expressing the coordinates $\tilde{y}$ as functions of the coordinates $y$ :

$$
\begin{aligned}
\tilde{y}_{1} & =\lambda^{-1}\left(\hat{x}_{1}-\hat{x}_{2}^{2}\right)=\lambda^{-1}\left(\tilde{x}_{1}-\tilde{x}_{2}^{2}\right) \\
& =\lambda^{-1}\left(\lambda x_{1}+\left(\lambda^{2}-\lambda\right) x_{2}^{2}+c_{1}-\lambda^{2} x_{2}^{2}\right) \\
& =x_{1}-x_{2}^{2}+\tilde{c}_{1}=y_{1}+\tilde{c}_{1} .
\end{aligned}
$$

Similarly, we get

$$
\begin{gathered}
\tilde{y}_{2}=\lambda^{-1} \hat{x}_{2}=\lambda^{-1} \tilde{x}_{2}=\lambda^{-1}\left(\lambda x_{2}\right)=x_{2}=y_{2} ; \\
\tilde{y}_{3}=e^{\hat{x}_{3}} \sin \hat{x}_{3}=e^{\tilde{x}_{3}+2 \pi} \sin \left(\tilde{x}_{3}+2 \pi\right)=e^{x_{3}} \sin x_{3}=y_{3}
\end{gathered}
$$

and

$$
\begin{aligned}
\tilde{y}_{4} & =e^{\hat{x}_{3}}\left(\sin \hat{x}_{3}+\cos \hat{x}_{3}\right) \hat{x}_{4} \\
& =e^{\tilde{x}_{3}+2 \pi}\left(\sin \left(\tilde{x}_{3}+2 \pi\right)+\cos \left(\tilde{x}_{3}+2 \pi\right)\right) \tilde{x}_{4} \\
& =e^{x_{3}} \sin x_{3}+e^{x_{3}} x_{4}^{2}=y_{4} .
\end{aligned}
$$

We conclude that the symmetries of the canonical form are exhausted here by a 1-parameter family of translations along the first variable. This is in concordance with the results in [38]. Notice that the composition $\Phi \circ \psi \circ \Phi^{-1}$ does not yield a symmetry for the canonical form. The reason is that, the system $\Sigma_{S F F}^{(q)}$, being defined around the equilibrium $q$, is not transformed into the canonical form $\Sigma_{S F C F}$ by the same diffeomorphism $\Phi$ as $\Sigma_{S F F}^{(o)}$ is. 
1. V. I. Arnold, Geometrical Methods in the Theory of Ordinary Differential Equations, Second Edition, Springer-Verlag, 1988.

2. A. Astolfi and F. Mazenc, A geometric characterization of feedforward forms, in Proc. MTNS'2000, Perpignan, France, 2000.

3. S. Battilotti, Semiglobal stabilization of uncertain block-feedforward forms via measurement feedback, in Proc. of NOLCOS'98, Enschede, the Netherlands, (1998), pp. 342-347.

4. V. D. Blondel and A. Megretski, Unsolved Problems in Mathematical Systems and Control Theory, Princeton University Press, 2004.

5. V. Bogaevski and A. Povzner, Algebraic Methods in Nonlinear Physics, Springer-Verlag, New York, 1991.

6. B. Bonnard, Quadratic control systems, Mathematics of Control, Signals, and Systems, 4 (1991), pp. 139160.

7. R. W. Brockett, Feedback invariants for nonlinear systems, in Proc. IFAC Congress, Helsinski, 1978.

8. M. Fliess, J. Lévine, P. Martin, and P. Rouchon, Flatness and defect of nonlinear systems: Introductory theory and examples, International Journal of Control, 6 (1995), pp. 1327-1361.

9. Hamzi, B., J.-P. Barbot and W. Kang (1998). Bifurcation and Topology of Equilibrium Sets for Nonlinear Discrete-Time Control Systems, Proc. of the Nonlinear Control Systems Design Symposium (NOLCOS'98), pp. 35-38.

10. Hamzi, B., J.-P. Barbot and W. Kang (1999). Normal forms for discrete time parameterized systems with uncontrollable linearization, Proceedings of the 38th IEEE Conference on Decision and Control, vol. 2, pp. 2035-2038.

11. Hamzi, B., J.-P. Barbot and W. Kang (1999). Bifurcation for discrete-time parameterized systems with uncontrollable linearization, Proc. of the 38th IEEE Conference on Decision and Control, vol.1, pp. 684 -688 .

12. Hamzi, B., J.-P. Barbot and W. Kang (1999). "Stabilization of Nonlinear Discrete-Time Control Systems with Uncontrollable Linearization". In Modern Applied Mathematics Techniques in Circuits, Systems and Control", World Scientific and Engineering Society Press, pp. 278-283. Also in Proc. of the 3rd IEEE/IMACS Multiconference on Circuits, Systems, Communications and Computers, pp. 4581-4586.

13. Hamzi, B., J.-P. Barbot, S. Monaco, and D. Normand-Cyrot (2001). Normal Forms versus Naimark-Sacker Bifurcation Control, invited paper to the Nonlinear Control Systems Design Symposium (NOLCOS'01).

14. Hamzi, B., J.-P. Barbot, S. Monaco, and D. Normand-Cyrot (2001). Nonlinear Discrete-Time Control of Systems with a Naimark-Sacker Bifurcation, Systems and Control Letters, 44, 245-258.

15. Hamzi, B, and Tall, I. A., Normal Forms for Nonlinear Discrete Time Control Systems, in CD Proceedings 42nd IEEE Conference on Decision \&S Control, 2003, pp. 1357-1361.

16. L. R. Hunt and R. Su, Linear equivalents of nonlinear time varying systems, in: Proc. MTNS, Santa Monica, CA, (1981), pp. 119-123.

17. A. Isidori, Nonlinear Control Systems, Second Edition, New York: Springer-Verlag, 1989.

18. B. Jakubczyk, Equivalence and Invariants of Nonlinear Control Systems, in Nonlinear Controllability and Optimal Control, H.J. Sussmann (ed.), Marcel Dekker, New York-Basel, (1990), pp. 177-218.

19. B. Jakubczyk and W. Respondek, On linearization of control systems, Bull. Acad. Polon. Sci. Ser. Math., 28 (1980), pp. 517-522.

20. B. Jakubczyk and W. Respondek, Feedback classification of analytic control systems in the plane, in: Analysis of Controlled Dynamical Systems, B. Bonnard et al. (eds.), Birkhäuser, Boston, (1991), pp. 262273.

21. M. Jankovic, R. Sepulchre, and P. Kokotovic, Constructive Lyapunov stabilization of nonlinear cascade systems, IEEE Trans. Automat. Control, 41 (1996), pp. 1723-1735.

22. W. Kang, Extended controller form and invariants of nonlinear control systems with single input, $J$. of Mathem. System, Estim. and Control, 4, (1994), pp. 1-25.

23. W. Kang, Quadratic normal forms of nonlinear control systems with uncontrollable linearization, Proc. 34th $C D C$, New Orleans, (1995).

24. W. Kang and A.J. Krener, Extended quadratic controller normal form and dynamic feedback linearization of nonlinear systems, SIAM J. Control and Optim., 30 (1992), pp. 1319-1337.

25. W. Kang, Bifurcation and normal form of nonlinear control systems - part I and II, SIAM J. Control and Optim., 36, (1998), 193-212 and 213-232.

26. W. Kang, Bifurcation control via state feedback for systems with a single uncontrollable mode, SIAM J. Control and Optim., 38, (2000), 1428-1452.

27. A.J. Krener, Approximate linearization by state feedback and coordinate change, Systems and Control Letters, 5 (1984), pp. 181-185.

28. A. J. Krener, S. Karahan, M. Hubbard, and R. Frezza, Higher order linear approximations to nonlinear control systems, Proc. of the 26 th CDC, Los Angeles, (1987) pp. 519-523. 
29. A. J. Krener, W. Kang, and D. E. Chang, Control bifurcations, IEEE Trans. on Automat. Control, 49 (2004), pp. 1231-1246

30. Krener, A.J. and L. Li (2002). Normal Forms and Bifurcations of Discrete Time Nonlinear Control Systems, SIAM J. on Control and Optimization, 40, 1697-1723.

31. A. Marigo, Constructive necessary and sufficient conditions for strict triangularizability of driftless nonholonomic systems,in Proc. 34th CDC, Phoenix, Arizona, USA, (1999), pp. 2138-2143.

32. F. Mazenc and L. Praly, Adding integrations, saturated controls, and stabilization for feedforward forms, IEEE Trans. Automat. Control, 41 (1996), pp. 1559-1578.

33. F. Mazenc and L. Praly, Asymptotic tracking of a reference state for systems with a feedforward structure, Automatica, 36 (2000), pp. 179-187.

34. R. Olfati-Saber, Fixed point controllers and stabilization of the cart-pole system and the rotating pendulum, Proc. of the 38th CDC, Phoenix, AZ, 1999, pp. 1174-1181.

35. R. Olfati-Saber, Normal forms for underactuated mechanical systems with symmetry, IEEE Trans. Automat. Control, 47 (2002), pp. 305-308.

36. W. Respondek, Feedback classification of nonlinear control systems in $R^{2}$ and $R^{3}$, in: Geometry of Feedback and Optimal Control, B. Jakubczyk and W. Respondek (eds.), Marcel Dekker, New York, (1998), pp. 347382.

37. W. Respondek, Transforming a single input system to a $p$-normal form via feedback, Proc. of the $42^{\text {nd }}$ IEEE CDC, Maui, Hawai, pp. 1574-1579.

38. W. Respondek and I. A. Tall, How Many Symmetries Does Admit a Nonlinear Single-Input Control System around Equilibrium, in Proc. of the 40th CDC, pp. 1795-1800, Florida, (2001).

39. W. Respondek and I.A. Tall, Nonlinearizable single-input control systems do not admit stationary symmetries, Systems and Control Lett., 46 (2002), pp 1-16.

40. W. Respondek and I.A. Tall, Strict feedforward form and symmetries of nonlinear control systems, in Proc. of the $43^{\text {rd }}$ IEEE Conf. on Decision and Control, Atlantis, Bahamas, pp. 1611-1616.

41. W. Respondek and I.A. Tall, Smooth and analytic normal and canonical forms for strict feedforward systems, Proc. of the 44th IEEE Conf. on Decision and Control, Seville, Spain, 2005, pp. 4213-4218.

42. W. Respondek and I. A. Tall, Feedback equivalence of nonlinear control systems: a survey on formal approach, to apper in Normal Forms, Bifurcations and Chaos in Automatic: From Theory Towards Applications, W. Perruquetti and J.-P. Barbot (eds.), Marcel Dekker, 2005.

43. W. Respondek and M.Zhitomirskii, Feedback classification of nonlinear control systems on 3-manifolds, Mathematics of Control, Signals and Systems, 8 (1995), pp. 299-333.

44. Sepulchre R., Janković M., and Kokotović P. Constructive Nonlinear Control, Springer, Berlin-HeidelbergNew York, 1996.

45. I. A. Tall, Normal Forms of Multi-Inputs Nonlinear Control Systems with Controllable Linearization, in New Trends in Nonlinear Dynamics and Control, and their Applications, W. Kang, M. Xiao, C. Borges (eds.), LNCS vol. 295, Springer, Berlin-Heidelberg, 2003, pp. 87-100.

46. I. A. Tall, Feedback Classification of Multi-Inputs Nonlinear Control Systems, submitted in SIAM Journal on Control and Optim..

47. I. A. Tall and W. Respondek, Feedback classification of nonlinear single-input control systems with controllable linearization: normal forms, canonical forms, and invariants, in SIAM Journal on Control and Optim., 41 (5), pp 1498-1531, (2003).

48. I. A. Tall and W. Respondek, Normal forms, canonical forms, and invariants of single-input control systems under feedback, Proc. 39th CDC, Sydney, (2000), pp. 1625-1630.

49. I. A. Tall and W. Respondek, Normal forms and invariants of nonlinear single-input systems with noncontrollable linearization, NOLCOS'01, Petersburg, Russia, (2001).

50. I.A. Tall and W. Respondek, Feedback equivalence to feedforward forms for nonlinar single-input control systems, in Dynamics, Bifurctions and Control, F. Colonius and L. Grüne (eds), LNCS vol. 273, Springer, Berlin-Heidelberg, 2002, pp. 269-286.

51. I. A. Tall and W. Respondek, Feedback Equivalence to a Strict Feedforward Form for Nonlinear SingleInput Systems, to appear in Int. Journal of Control.

52. I. A. Tall and W. Respondek, Transforming a Single-Input Nonlinear System to a Strict Feedforward Form via Feedback, Nonlinear Control in the Year 2000, A. Isidori, F. Lamnabhi, and W. Respondek, (eds.), Springer-Verlag, 2, pp. 527-542, London, England, (2001).

53. I. A. Tall and W. Respondek, Feedback Equivalence to Feedforward Form for Nonlinear Single-Input Systems, Dynamics, Bifurcations and Control, F. Colonius and L. Grune (eds.), LNCIS, 273, pp. 269-286, Springer-Verlag, Berlin Heidelberg, (2002).

54. I.A. Tall and W. Respondek, Transforming a single-input nonlinear system to a feedforward form via feedback, in Nonlinear Control in the Year 2000, A. Isidori, F. Lamnabhi-Lagarrigue, and W. Respondek (eds.), Springer, 2 (2000), pp. 527-542.

55. I.A. Tall and W. Respondek, Weighted Canonical Forms, inProc. 43rd IEEE Conference on Decision and 
Control, Atlantis, Bahamas (2004), pp. 1617-1622.

56. I. A. Tall and W. Respondek, Smooth and analytic normal forms: A special class of strict feedforward forms, in Control and Observer Design in Nonlinear Finite and Infinite Dimensional Systems, Th. Meurer, K. Graichen E.D. Gilles (eds.), LNCIS, 322, Springer-Verlag, Berlin Heidelberg, (2005).

57. I. A. Tall and W. Respondek, Explicit Symmetries of Strict Feedforward Control Systems, Proc. 45th IEEE Conference on Decision and Control, San Diego, USA (2006), pp. 3813-3818.

58. D.G. Taylor and S. Li, stable inversion of continous-time nonlinear systems by finite-difference methods, IEEE Trans Autom Control, 47 (2002), pp. 537-542.

59. A. Teel, Feedback stabilization: nonlinear solutions to inherently nonlinear problems, Memorandum UCB/ERL M92/65.

60. A. Teel, A nonlinear small gain theorem for the analysis of control systems with saturation, IEEE Trans Autom Control, 41 (1996), pp. 1256-1270.

61. M. Zeitz, Controllability canonical (phase-variable) form for non-linear time-variable systems, Int. J. Control, 37 ( 1983), pp. 1449-1457.

62. M. Zeitz, Canonical forms for nonlinear systems, Nonlinear Control Systems Design, A. Isidori (ed.), IFAC-Symp., Pergamon Press, Oxford, 1989, pp. 33-38.

63. M. Zhitomirskii and W. Respondek, Simple germs of corank one affine distributions, Banach Center Publications, 44, (1998), 269-276. 